\title{
Persistently laminar branched surfaces
}

\author{
YING-QING WU
}

\begin{abstract}
We define sink marks for branched complexes and find conditions for them to determine a branched surface structure. These will be used to construct branched surfaces in knot and tangle complements. We will extend Delman's theorem and prove that a non2-bridge Montesinos knot $K$ has a persistently laminar branched surface unless it is equivalent to $K\left(1 / 2 q_{1}, 1 / q_{2}, 1 / q_{3},-1\right)$ for some positive integers $q_{i}$. In most cases these branched surfaces are genuine, in which case $K$ admits no atoroidal Seifert fibered surgery. It will also be shown that there are many persistently laminar tangles.
\end{abstract}

\section{Introduction}

Essential lamination plays an important role in the study of topology of 3-manifolds and exceptional Dehn surgery. Denote by $K(r)$ the manifold obtained by $r$ surgery on a hyperbolic knot $K$ in a closed 3-manifold. A nontrivial surgery is exceptional if $K(r)$ is non-hyperbolic, i.e., it is reducible, toroidal or Seifert fibered. A 3-manifold is laminar if it contains an essential lamination. If $M$ is laminar then it is irreducible, and if the lamination is genuine in the sense that some complementary region is not an $I$-bundle then $M$ is not a small Seifert fibered manifold. In certain cases essential laminations can also be used to detect toroidal manifolds, see for example [11].

A lamination is essential if and only if it is carried by an essential branched surface [6]. In [8] Li defined laminar branched surfaces. These are essential branched surfaces that satisfy some mild extra conditions, which implies that it carries a lamination, hence an essential lamination. Denote by $K(r)$ the manifold obtained by Dehn surgery on a knot $K$ along slope $r$. A laminar branched surface $\Sigma$ in the exterior of $K$ is persistently laminar if it remains laminar in $K(r)$ for all non-meridional slopes $r$.

Combinatorially a branched surface is a 2-complex $\Sigma$ whose singular set $b(\Sigma)$ is a set of immersed curves on $\Sigma$, called the branch curves or branch loci of $\Sigma$. A branched surface structure on $\Sigma$ is a smooth structure in a neighborhood of $b(\Sigma)$ so that every point has a neighborhood modeled on 


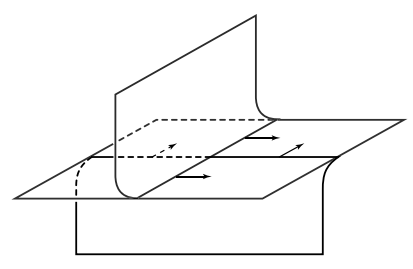

(a)

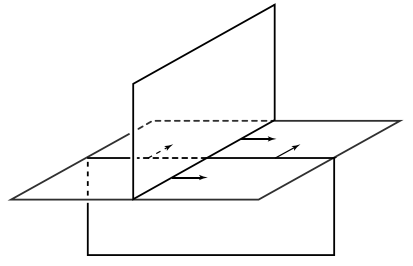

(b)

Figure 1

that in figure 1(a). Li used sink directions on $b(\Sigma)$ to indicate the smooth structure near $b(\Sigma)$. See Section 2. This is a very useful way to define a branched surface structure on $\Sigma$; however, it is difficult to use when the branched surface is complicated. Since there are only three ways to smooth the complex near any branch curve, we can indicate the smooth structure on a segment $\alpha$ of $b(\Sigma)$ using a sink mark instead, which is either an orientation or a diamond sign on $\alpha$. See Section 3 for more details. This is particularly useful for tangle complexes $\Sigma=Q \cup P \cup D$ in the exterior of a tangle or knot $L$, where $Q$ is the tube on the boundary of a tubular neighborhood of $L, P$ is a set of punctured surfaces with $\partial P$ a set of meridional curves on $Q$, and $D$ a set of surfaces with boundary on $P \cup Q$ intersecting each meridian of $Q-P$ exactly once. The Unique Extension Lemma (Lemma 3.7) shows that the branched surface structure of such a complex is completely determined by the sink marks on $\partial D$, and these sink marks define a branched surface structure if and only if they satisfy some simple conditions.

We will use this result to reconstruct Delman's channel surfaces [4]. By Li's result [8] and Lemma 3.7 it is now easy to show that these branched surfaces are laminar, and most of them are genuine. See Theorem 5.3. In Section 6 we will further explore and extend Delman's half channel surfaces construction using sink marks. The simple pictures of sink marks on these branched surfaces allow us to find various semi-allowable paths with desirable properties in the Hatcher-Thurston diagram of a rational tangle. See Proposition 6.5. We can then strengthen the main theorem of Delman in [4], which says that a Montesinos knot has a persistent lamination unless it is a pretzel knot $K=K\left(p_{1} / q_{1}, p_{2} / q_{2}, p_{3} / q_{3}, n\right)$, where $p_{i}=1$ or $q_{i}-1, q_{1}$ is even, $q_{i}$ positive, and $n=-1$ or -2 . The following theorem shows that up to equivalence (i.e., up to taking mirror image) we must have $p_{i}=1$ and $n=-1$. 
Theorem 6.6. Let $K$ be a non 2-bridge Montesinos knot. Then $K$ has a persistently laminar branched surface in its complement unless it is equivalent to $K\left(1 / q_{1}, 1 / q_{2}, 1 / q_{3},-1\right)$, where $q_{i}$ are positive integers, and $q_{1}$ is even.

As a consequence, we see that the knot $10_{142}$ is persistently laminar. This is one of the 5 knots with crossing number at most 10 which were not known whether surgery on them always produce laminar manifolds $[5$, FQ 1.2]. The construction can also be applied to more general knots. For example, if $F$ is a minimal Seifert surface of a two-component link $L, \alpha$ is an arc on $F$ connecting the two components, and $K$ is obtained by replacing $N(\alpha) \cap L$ with a $2 n$-twist tangle with $|n| \geq 2$, then $K$ is persistently laminar. See Corollary 6.9.

We are particularly interested in determining which Montesinos knots of length 3 have a persistently laminar branched surface which is genuine in the sense that it has a complementary region which is not an $I$-fiber. The following shows that most of them do have such a branched surface.

Theorem 6.7. Let $K$ be a Montesinos knot of length 3. Then $K$ has a genuine persistently laminar branched surface in its complement unless $K$ is equivalent to $K\left(1 / q_{1}, 1 / q_{2}, p_{3} / q_{3}, n\right)$, such that either

(1) $n=0, q_{i} \geq 2$, and $p_{3}=1$; or

(2) $n=-1, q_{i} \geq 2$, and $p_{3}=1,2$ or $q_{3}-1$.

Exceptional surgeries on arborescent knots have all been classified except atoroidal Seifert fibered surgeries on Montesinos knots of length 3. By a theorem of Brittenham [1], if $K$ has a genuine persistently laminar branched surface then $K(r)$ is not a small Seifert fibered manifold for any nontrivial $r$. Using this and the results of [12] we have the following two theorems, according to whether $K$ is pretzel or not. Here a Montesinos knot is a pretzel $k n o t$ if it can be written as $K\left(1 / q_{1}, 1 / q_{2}, \ldots, 1 / q_{k}, n\right)$ for some integers $q_{i}$ and $n$ with $\left|q_{i}\right| \geq 2$, and it is a genuine pretzel knot if in addition $n=0$. The number $k$ is called the length of $K$.

Theorem 7.2. Let $K$ be a pretzel knot of length 3. If $K$ admits an atoroidal Seifert fibered surgery, then $K$ is equivalent to $K\left(\frac{1}{q_{1}}, \frac{1}{q_{2}}, \frac{1}{q_{3}}, n\right)$ such that either $n=0$ and hence $K$ is a genuine pretzel knot, or $n=-1$ and $q_{i} \geq 3$. In either case the $q_{i}$ satisfy $\frac{1}{\left|q_{1}\right|-1}+\frac{1}{\left|q_{2}\right|-1}+\frac{1}{\left|q_{3}\right|-1}>1$. 
Note that $q_{i}$ satisfies the above inequality if and only if, up to relabeling, $\left(\left|q_{1}\right|,\left|q_{2}\right|,\left|q_{3}\right|\right)=\left(2,\left|q_{2}\right|,\left|q_{3}\right|\right),\left(3,3,\left|q_{3}\right|\right)$ or $(3,4,5)$.

Theorem 7.3. Let $K$ be a Montesinos knot of length 3. If $K$ is not a pretzel knot and $K$ admits an atoroidal Seifert fibered surgery $K(r)$, then $K$ is equivalent to one of the following:

(a) $K(-2 / 3,1 / 3,2 / 5)$;

(b) $K(-1 / 2,1 / 3,2 /(2 a+1))$ and $a \in\{3,4,5,6\}$;

(c) $K(-1 / 2,1 / q, 2 / 5)$ for some $q \geq 3$ odd.

The construction in Section 5 can also be modified to make persistently laminar branched surfaces in tangle spaces. A 2-string tangle $(B, T)$ is persistently laminar if $B-T$ contains a branched surface $\Sigma$, such that if $K$ is any knot that can be written as the union of $(B, T)$ with another tangle $\left(B^{\prime}, T^{\prime}\right)$, which is non-trivial in the sense that a curve of slope 0 on $\partial B$ does not bound a disk in $B^{\prime}-T^{\prime}$, then $\Sigma$ is a persistently laminar branched surface for $K$. Brittenham [1] showed that the tangle $T(1 / 3,-1 / 3)$ is persistently laminar. In [13] Youn proved that the tangle $T(1 / 3,-1 / 5)$ is also persistently laminar. A Montesinos tangle of length 2 is homeomorphic to some $T\left(r_{1},-r_{2}\right)$ with $0<r_{i}<1$ and $r_{1}+r_{2} \leq 1$. The following theorem shows that many of these are persistently laminar tangles if both $q_{i}$ are odd.

Theorem 8.5. If $0<r_{i}=p_{i} / q_{i}<\frac{2}{3}$ and $q_{i}$ are odd then $T=T\left(r_{1},-r_{2}\right)$ is persistently laminar.

Thus for example, assuming $r_{i}=p_{i} / q_{i}$ and $q_{i} \geq 3$ are odd then all tangles of type $T\left(1 / q_{1}-1 / q_{2}\right)$ or $T\left(r_{1},-r_{1}\right)$ are persistently laminar, and if $r_{2} \in$ $\left(\frac{1}{3}, \frac{2}{3}\right)$ then $T\left(r_{1},-r_{2}\right)$ is persistently laminar for all $r_{1} \in(0,1)$ satisfying the above conditions. See Example 8.6 for more details.

The paper is organized as follows. Section 2 gives some basic definitions and lemmas, including laminar, pre-laminar and combinatorial branched surfaces, which is a branched 2-complex $\Sigma$ with sink directions assigned, satisfying certain combinatorial conditions. Proposition 2.4 shows that these conditions determine a unique branched surface structure on $\Sigma$. Section 3 introduces sink marks and tangle complexes, and proves the Unique Extension Lemma 3.7, which gives an easy way to detect pre-laminar branched surfaces among tangle complexes with sink marks. Section 4 constructs Hatcher-Thurston branched surfaces corresponding to any edge path in the Hatcher-Thurston diagram. It will be used later in the constructions of other 
branched surfaces. Section 5 defines Delman channel and Delman channel surfaces $\Sigma$ corresponding to any allowable path $\gamma$, proves Delman's theorem that such a branched surface in a knot complement is persistently laminar and shows that $\Sigma$ is genuine if some vertex of $\gamma$ has corner number at least 3. A familiarity with the earlier constructions of Hatcher-Thurston and of Delman will be helpful in understanding Sections 4 and 5. In Section 6 we extend Delman's construction of half channel branched surfaces and prove an existence result of semi-allowable paths with certain properties (Proposition 6.5), which is then used to prove the existence theorems (Theorems 6.6 and 6.7 ) for persistently laminar branched surfaces. These are applied in Section 7 to study atoroidal Seifert fibered Dehn surgery. Section 8 constructs persitently laminar branched surfaces in tangle spaces.

All manifolds are assumed compact, connected and orientable unless otherwise stated. For any submanifold $Y$ in $X$, denote by $X \mid Y$ the manifold obtained by cutting $X$ along $Y$. When a rational number $r$ is written as $p / q$ it is always assumed that $p, q$ are coprime.

\section{Combinatorial description of branched surfaces}

We refer the readers to [6] for basic definitions such as essential lamination, essential branched surface $F$, its regular neighborhood $N(F)$, its $I$-fibers, the collapsing map $\pi: N(F) \rightarrow F$, the horizontal surface $\partial_{h} F$ and the vertical surface $\partial_{v} F$. The vertical surface $\partial_{v} F$ is also called the cusps of $F$. Recall that the branch locus $b(F)$ of a branched surface $F$ is the set of points which does not have a disk neighborhood. It is a finite union of immersed curves on $F$. The set of double points of $b(F)$ is denoted by $s(F)$, called the singular points of $F$, which cuts $b(F)$ into arcs and circles, called the branch curves. A point in $b(F)-s(F)$ has a neighborhood which is the union of three disks $F_{1}, F_{2}, F_{3}$ joined at an arc in $b(F)-s(F)$, and a point in $s(F)$ has a neighborhood modeled in figure 1(a). Li [8] uses an arrow to indicate the sink direction of $\alpha$. It is an arrow pointing from $\alpha$ into one of the surfaces attached to it, so that if the vertical surface of a regular neighborhood of $F_{1} \cup F_{2} \cup F_{3}$ lies between $F_{2}$ and $F_{3}$ then the sink direction will point from $\alpha$ into $F_{1}$, as shown in figure $1(\mathrm{a})$. We say that $\alpha$ is a sink edge of $F_{1}$ and a source edge of $F_{2}$ and $F_{3}$. We will also consider $\alpha$ as a cusp on the side of $F_{2} \cup F_{3}$ opposite to the surface $F_{1}$ since it corresponds to a piece of the cusps $\partial_{v} F$ on that side. The introduction of sink direction greatly simplifies the way to draw branched surfaces since now we only need to draw the topological 2-simplex and the sink directions and do not have to draw it 
with the actual tangency or smooth structure. For example, we can use the 2-complex in figure 1(b) to denote the branched surface in figure 1(a).

The branch locus $b(F)$ cuts $F$ into several surfaces, called the branches of $F$. We allow a branched surface $F$ to have non-empty boundary $\partial F$, which is a train track. Thus the boundary of a branch of $F$ is a union of sink arcs, source arcs, and possibly some arcs on $\partial F$. A disk branch $D$ of $F$ is called a sink disk if $\partial D$ contains some sink edges but no source edge. This matches the definition in [8] when $F$ has no boundary. Similarly, a disk is a source disk if it has source edges but no sink edge, and a passing disk if it contains both sink edges and source edges. $F$ is sinkless if it contains no sink disk.

A cusped manifold is a pair $(M, \gamma)$, where $M$ is a compact orientable 3 -manifold, and $\gamma$ is a set of simple closed curves on $\partial M$. Denote by $\partial_{v}(M)$ a regular neighborhood of $\gamma$ on $\partial M$, called the vertical boundary, and by $\partial_{h}(M)$ the surface $\partial M-\operatorname{Int}_{v}(M)$, called the horizontal surface. If $\Sigma$ is a branched surface in a 3-manifold $Y$, we use $E(\Sigma)$ to denote $Y-\operatorname{Int} N(\Sigma)$, called the exterior of $\Sigma$. It is a cusped manifold with $\gamma$ the central curve of $\partial_{v}(\Sigma)$, so $\partial_{h}(M)=\partial_{h}(\Sigma)$ and $\partial_{v}(M)=\partial_{v}(\Sigma)$. A disk $D$ in $M$ is a monogon if $\partial D$ intersects $\gamma$ transversely at a single point. A cusped manifold $(M, \gamma)$ is essential if $M$ is irreducible, has no monogon, and $\partial_{h} M$ is incompressible and has no sphere component. If $M$ is a solid torus, the cusp winding number of $(M, \gamma)$ is the minimal intersection number between $\gamma$ and a meridian of $M$. It is easy to see that in the definition of essentiality of $M$, the condition that $M$ has no monogon can be replaced by the weaker condition that no component of $M$ is a solid torus with cusp winding number 1 .

A surface carried by $\Sigma$ is an embedded surface in $N(\Sigma)$ transverse to the $I$-fibers. Let $\pi: N(\Sigma) \rightarrow \Sigma$ be the collapsing map, which maps each $I$-fiber to a single point of $\Sigma$.

Definition 2.1. (1) A branched surface $\Sigma$ is atoroidal if any torus carried by $\Sigma$ is parallel to a component of $\partial_{h} \Sigma$ in $N(\Sigma)$.

(2) An embedded sphere $S$ in $\Sigma$ is a trivial bubble if one side of $S$ has no branch attached. In this case $S$ is parallel to a spherical component $S^{\prime}$ of $\partial N(F)$ such that $\pi: S^{\prime} \rightarrow \Sigma$ is injective.

(3) A branched surface $\Sigma$ is pre-laminar if it is sinkless, atoroidal and has no trivial bubble.

(4) A closed branched surface $\Sigma$ embedded in a closed 3-manifold $M$ is laminar if it is sinkless, it has no trivial bubble, it carries no Reeb torus (i.e. a torus which bounds a solid torus in $M)$, and $E(\Sigma)$ is an essential cusped manifold. 
(5) A branched surface $\Sigma$ in a 3-manifold $M$ with $\partial \Sigma \subset \partial M$ is genuine if at least one component $Y$ of $E(\Sigma)$ in the interior of $M$ is not an $I$-bundle with $\partial_{h} Y$ the corresponding $\partial I$-bundle.

Li [8, Theorem 1] proved that a laminar branched surface in a closed orientable 3-manifold carries an essential lamination and hence is an essential branched surface. A laminar branched surface does not have to be pre-laminar because it may be toroidal. Neither is a pre-laminar branched surface in a closed 3-manifold $M$ necessarily laminar. Being pre-laminar is an intrinsic property and is independent of the embedding of $\Sigma$ in a 3-manifold, hence it can be determined before it is embedded in $M$. The following result follows from [8, Theorem 1] immediately since a pre-laminar branched surface carries no torus and hence no Reeb torus.

Lemma 2.2. If $\Sigma$ is a closed pre-laminar branched surface, then its embedding in a compact orientable 3-manifold $M$ is laminar (and hence essential) if and only if $E(\Sigma)$ is an essential cusped manifold.

We note that the above lemma works for closed branched surface only. To extend it to branched surfaces with boundary we need to modify the definition of pre-laminar branched surfaces and essential cusped manifold, for example there should be no trivial half-bubble on $\Sigma$ and the horizontal surface of $E(\Sigma)$ must be boundary incompressible. In the constructions below we will need to construct pre-laminar branched surfaces with boundary, but these will eventually be combined to make closed branched surfaces. Therefore we only need the above lemma when $\Sigma$ is a closed branched surface.

Using sink directions we can define a branched surface combinatorially.

Definition 2.3. (1) A compact 2-complex $F$ is a branched complex if it is locally modeled on the 2-complex in figure 1(b) up to homeomorphism (without sink arrows specified). Denote by $b(F)$ the set of points which do not have a surface neighborhood, and by $s(F)$ set of points in $b(F)$ which does not have an arc neighborhood in $b(F)$, called the singular set of $F$.

(2) A sink direction on a component $\alpha$ of $b(F)-s(F)$ is a vector pointing from $\alpha$ into one of the three incidented surfaces.

(3) A combinatorial branched surface is a branched complex $F$ with a sink direction assigned for each component of $b(F)-s(F)$, such that for each point $p \in s(F)$, the $4 \operatorname{arcs} e_{1}, \ldots, e_{4}$ in $b(F)$ and the $6 \operatorname{disks} A_{i j}(1 \leq i<j \leq$ 4 ) in a neighborhood of $p$ can be labeled so that $A_{i j} \cap b(F)=e_{i} \cup e_{j}, A_{12}$ is a sink disk, $A_{2,3}, A_{1,4}$ are passing disks, and the others are source disks. 
The following proposition shows that a combinatorial branched surface is a branched surface after smoothing along the branch loci according to the sink directions. We will therefore consider any combinatorial branched surface as a branched surface, and vise versa.

Proposition 2.4. Any combinatorial branched surface $F$ is homeomorphic to a branched surface $F^{\prime}$ with sink directions preserved. If $F$ is embedded in a 3-manifold $M$ then it is isotopic to a branched surface with sink directions preserved.

Proof. We assume $F \subset M$. The other case is similar. There is no problem smoothing $F$ along $b(F)-s(F)$ according to the sink direction, so we need only verify that the neighborhood of a point $p \in s(F)$ can be deformed to a branched surface according to the sink directions. Let $D$ be the disk $A_{12} \cup A_{23} \cup A_{34} \cup A_{14}$. By definition $A_{12}$ is a sink disk, $A_{34}$ is a source disk, and the other two are passing disks; hence each $e_{i}$ is a sink edge of one disk and a source edge of another in $D$, so up to isotopy we may assume $D$ is a smooth disk in $M$, and $c^{\prime}=e_{1} \cup e_{3}$ and $c^{\prime \prime}=e_{2} \cup e_{4}$ are smooth arcs on $D$.

Note that each branch curve is a sink edge of exactly one of the three incidented disks. The disks $A_{13}$ intersect $D$ at $c^{\prime}=e_{1} \cup e_{3}$. Since the sink directions of $e_{1}, e_{3}$ points into $A_{12}$ and $A_{23}$ respectively, which are on the same side of $c^{\prime}$, the tangency of $A_{13}$ along $e_{1}, e_{3}$ matches at $p$, so $D \cup A_{13}$ is a branched surface. Since $A_{24}$ and $A_{13}$ has disjoint interior while their boundaries intersect transversely at $p$, they must lie on different sides of $D$. For the same reason, the sink directions on the two boundary edges $e_{2}, e_{4}$ of $c^{\prime \prime}$ points to the same side of $c^{\prime \prime}$, so the smoothing along these two edges matches at $p$, hence $D \cup A_{13} \cup A_{24}$ is a branched surface after smoothing.

Remark 2.5. (1) The sink directions define a branched surface structure in a neighborhood $X$ of $p \in s(F)$ if and only if (i) there is exactly one sink disk, two passing disks and three source disks, and (ii) the two passing disks intersect only at $p$, and the sink directions of their source edges point into the same disk. Thus among the $4^{3}=81$ possible choices of sink directions, only 12 of them make $X$ a branched surface.

(2) The sink directions of a branched surface near a singular point $p$ is completely determined by those of the two passing disks in a neighborhood of $p$.

Example 2.6. The 2-simplices in figures 2(a), (b) and (c) are not branched surfaces. The surface in (a) has two sink disks, the one in (b) has no sink disk, 


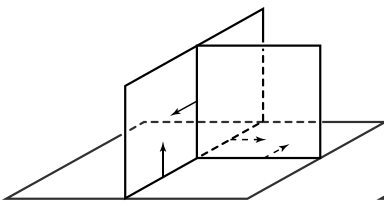

(a)

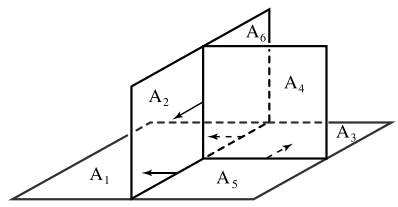

(d)

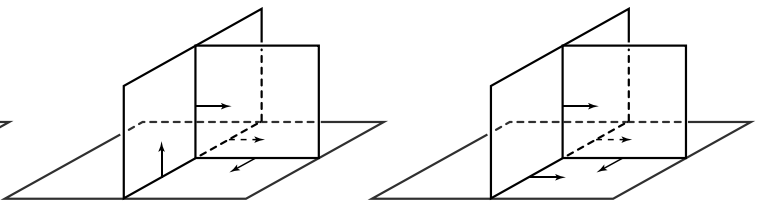

(c)

(b)

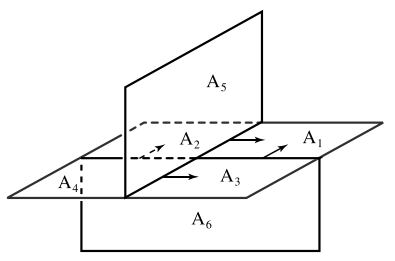

(e)

Figure 2

and in (c) the two passing disks have one edge in common, which implies that it is not a branched surface by Remark 2.5. One can check that the 2-complex in figure 2(d) satisfies Definition 2.3(3) and hence is a branched surface. It is homeomorphic to that in figure $2(\mathrm{~d})$, which is the same as the branched surface in figure $1(\mathrm{~b})$.

\section{Sink marks for branched surfaces}

While sink directions make it possible to define branched surfaces combinatorially, practically it is still very difficult using it to define branched surface structure on branched complex with more than just a very few branch curves. We need to further simplify it in order to use it to illustrate the branched surfaces to be constructed. Since each branch curve $\alpha$ has three possible sink directions, we can use an orientation of $\alpha$ and a diamond sign to indicate such a choice.

More explicitly, define a surface decomposition of a 2-complex $\Sigma$ to be a set of compact surfaces $S_{1}, \ldots, S_{n}$ in $\Sigma$ with mutually disjoint interiors, such that $\cup S_{i}=\Sigma$, and each component $\alpha$ of $b(\Sigma)-s(\Sigma)$ is on the boundary of one $S_{i}$ and the interior of another $S_{j}$. Thus in a neighborhood of $\alpha, \Sigma$ is obtained by attaching $S_{i}$ to $S_{j}$ along the branch curve $\alpha$.

Definition 3.1. Suppose $\left\{S_{i}\right\}$ is a surface decomposition of $\Sigma$. Then a sink mark on $\alpha \subset \partial S_{i} \cap$ Int $S_{j}$ is a diamond sign or an orientation of $\alpha$. The edge 


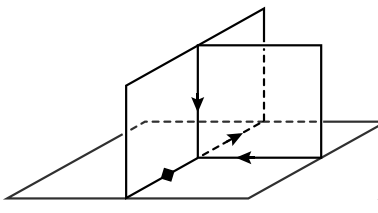

(a)

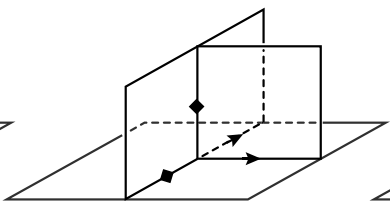

(b)

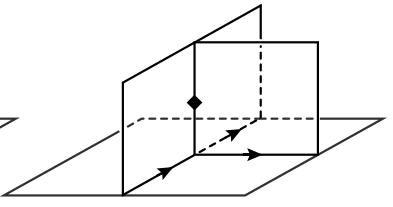

(c)

Figure 3

$\alpha$ is a called a diamond edge or an oriented edge accordingly. It determines a sink direction on $\alpha$ as follows.

(1) The sink direction of a diamond edge on $\partial S_{i}$ points into $S_{i}$.

(2) The orientation of an oriented edge $\alpha \subset \partial S_{i}$ defines a local orientation of $S_{i}$ and the sink direction points to the side of positive normal direction of $S_{i}$. When $M=S^{3}$ we use the orientation convention that the sink direction points to the right of $\alpha$ when standing on $S_{j}$ on the side of $S_{i}$ and facing to the direction of the orientation of $\alpha$.

(3) A set of sink marks, one for each branch curve, defines a branched surface structure on $X$ if $X$, with sink directions determined by the sink marks, is a branched surface.

For example, the 2-complexes in figures 2(a)-(c) have natural surface decompositions $\left(S_{1}, S_{2}, S_{3}\right)$ with each $S_{i}$ a flat disk. The corresponding sink marks are given in figures $3(\mathrm{a})-(\mathrm{c})$, respectively. Note that when a branched surface $\Sigma \subset S^{3}$ is mapped to $\Sigma^{\prime}$ by an orientation reversing map of $S^{3}$ then all the orientation marks of the branch curves are reversed because the global orientation has reversed.

A curve $C$ with several segments marked by sink marks is consistently oriented if it has an orientation which matches all sink marks on it. In particular, it has no diamond marks. For example, the central circle in figures $4(3)$ is consistently oriented while those in figures $4(1)-(2)$ are not. Also, three of the six rectangles in figures $4(1)-(3)$ are consistently oriented.

Now consider the branched complex $X$ shown in figure 4(1). It has an obvious surface decomposition $(Q, P, D)$, where $Q$ is the vertical annulus, $P$ the horizontal annulus with inner boundary attached to $Q$, and $D=$ $D_{1} \cup D_{2}$ is a pair of disks, such that each of $\alpha_{i}=D_{i} \cap P$ and $\beta_{i}=D_{i} \cap Q$ is a single arc.

Definition 3.2. When both segments of $\partial P$ are diamond edges as shown in figure $4(2), \partial P$ is called a meridional cusp of $X$. Note that in this case the cusp corresponding to $\partial P$ is on the inside side of $Q$. 


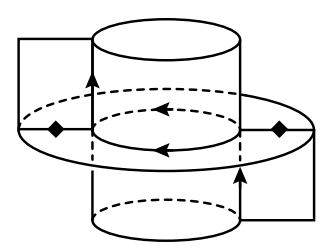

(1)

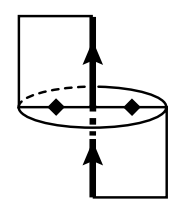

(I)

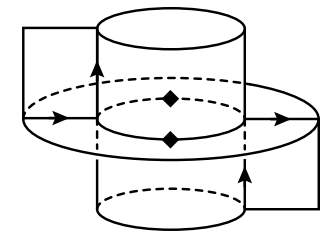

(2)

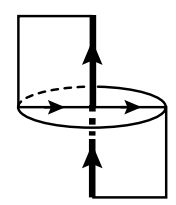

(II)

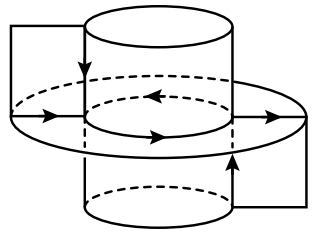

(3)

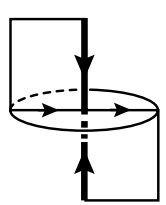

(III)

Figure 4

Definition 3.3. Let $X$ be the underlying 2-complex in figures 4(1) with surface decomposition $(Q, P, D)$ as above. A set of sink marks assigned on the four edges of $\partial D$ (but not on $\partial P$ ) is of type (I) if $\partial D \cap P$ are diamond edges and the two edges of $\partial D \cap Q$ on $Q$ point to the same direction, of type (II) if $\partial D$ has no diamond edge and each $\partial D_{i}$ is consistently oriented, and of type (III) if $\partial D$ has no diamond edge and exactly one $\partial D_{i}$ is consistently oriented.

Thus up to homeomorphism of $X, \partial D$ is as shown in figures 4(1)-(3) respectively, with possibly the orientations of both segments of $\partial D_{i}$ reversed for one or both $D_{i}$ if it is of type (II) or (III). Since there is no specification for sink marks on $\partial P$, we can shrink $Q$ and use a thick arc $K$ to represent $Q$ without loss of sink mark information, so the $X$ with sink marks in figures 4(1)-(3) can be represented by those in figures 4(I), (II) and (III), respectively. The sink marks on $\partial D$ induces a piecewise orientation on $K$, called the induced orientation, or the orientation induced by the sink marks. The following result shows that these sink mark systems can be uniquely extended over $\partial P$ to define a branched surface structure on $X$.

Lemma 3.4. Let $X=Q \cup P \cup D$ be as above. Then a sink mark system on $\partial D$ with no diamond on $\partial D \cap Q$ can be extended to a branched surface structure of $X$ if and only if it is of type (I), (II) or (III), in which case the 
extension is unique. In particular, $\partial P$ is a meridional cusp if and only if $X$ is of type (II).

Proof. Let $D_{1}$ be the upper left disk and $D_{2}$ the lower right disk of $D$ in figures 4(1)-(3). One can check that the sink directions corresponding to the sink marks in figures 4(1)-(3) satisfy the conditions in Definition 2.3(3), hence determine a branched surface structure in each case. Therefore the extensions exist. To prove the uniqueness, assume $X$ has been assigned a sink mark system so that $X$ is a branched surface. There are three possibilities for the sink marks on $\partial P$. We want to show that each of them corresponds to one of the types above, and the sink marks on $\partial P$ are completely determined by those on $\partial D$.

Case 1. One edge of $\partial P$ has a diamond mark. In this case this edge has a cusp on the inside of $Q$, which must extend to the other edge on $\partial P$ because by assumption $\partial D \cap Q$ are not diamond edges. Thus both edges of $\partial P$ are diamond edges. In this case $\partial D_{i}$ does not pass the cusp, hence must be consistently oriented, as shown in figure 4(2), possibly with orientations of both segments of $\partial D_{i}$ reversed for one or both $i$. Therefore $X$ is of type (II).

Case 2. The two segments of $\partial P$ are inconsistently oriented. In this case, near each singular point of $X$ the branch on $Q$ with interior disjoint from $D$ is a passing disk, hence by Remark 2.5(2), if these sink marks defines a branched surface structure then the sink marks on $\partial D$ are completely determined by those on $\partial P$. Since the ones in figure 4(1) do define a branched surface structure, it follows that the sink marks on $\partial D$ must be as in figure 4(1) if $\partial P$ is oriented as in the figure. Similarly if the orientations of both segments of $\partial P$ are reversed then the sink marks on $\partial D$ are obtained by reversing the orientations of $\partial D \cap Q$. In either case $X$ is of type $\mathrm{I}$. Note that the orientations on $\partial P$ are also determined by the sink marks on $\partial D$ as they have to point to the disk $D_{i}$ such that $D_{i} \cap Q$ has a tail at $\partial D_{i} \cap \partial P$.

Case 3. $\partial P$ is consistently oriented. Assume that the orientation of $\partial P$ is as shown in figure 4(3), then the cusp on $\partial P$ is on the upper side of $P$. There are two ways to attach each $D_{i} . \partial D_{1}$ passes through the cusp and must have inconsistent orientations on the two segments, and the orientations on $\partial D_{2}$ are consistent since it does not pass a cusp. Therefore $X$ is of type (III). Similarly if the orientation of $\partial P$ is reversed then $\partial D_{1}$ is consistently oriented while $\partial D_{2}$ is inconsistently oriented. Hence the orientation of $\partial P$ is determined by the sink marks on $\partial D$ according to the fact that the cusp at $\partial P$ is on the side of the disk $D_{i}$ whose boundary is inconsistently oriented. 


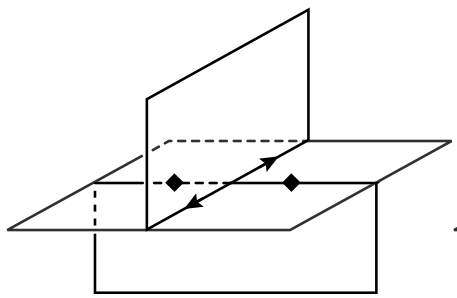

(a)

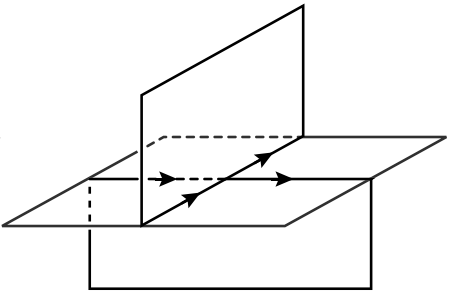

(b)

Figure 5

We have shown that the three possibilities above correspond to the three types of $X$ and in each case the sink marks on $\partial P$ are also completely determined by those on $\partial D$. This completes the proof of the lemma.

Definition 3.5. A tangle complex is a branched complex $X$ in $S^{3}$ with surface decomposition $(Q, P, D)$ as follows. Suppose $K \subset S^{3}$ is a compact 1-manifold and $S$ is a set of spheres such that $S_{i} \cap K \neq \emptyset$ for any component $S_{i}$ of $S$. Let $N(K)=K \times D^{2}$ be a tubular neighborhood of $K, Q$ the tubes $K \times \partial D^{2}, P$ the punctured spheres $S$ - Int $N(K)$, and $D$ a set of compact surfaces attached to $Q \cup P$ along some boundary curves $\gamma$ of $D$. They satisfy (i) each component of $\partial P$ is either a component of $\partial Q$, or it has a regular neighborhood modeled on the underlying 2-complex in figure 4(1); (ii) each meridian curve of $Q-P$ intersects $D$ at exactly one point, and (iii) each component of $D$ has some boundary arcs on $P$ and some on $Q$.

Lemma 3.4 allows us to use a thick curve to represent a tube $Q$ when a tangle complex is a branched surface because there is no need to specify the sink marks on $\partial P$, as long as each point of $K \cap S$ has a neighborhood of type (I), (II) or (III) as shown in figure 4 . Thus when drawing $X$, we will simply draw $(K, S, D)$, with the understanding that the thickened curve $K$ represents the tube $Q$ around $K$, and a disk transverse to $K$ in the figures represents a punctured sphere $P$.

A point $p$ in the singular set $s(X)$ of a tangle complex $X=Q \cup P \cup D$ is either on $\partial P$ or in the interior of $P$. In the latter case the neighborhood of $p$ consists of one subdisk $P_{0}$ on $P$ and two subdisks $D_{1}, D_{2}$ of $D$, one attached on each side of $P_{0}$. See figure 5 .

Definition 3.6. Let $\Sigma=Q \cup P \cup D$ be a tangle complex with sink marks specified on $\partial D$. Then a singular point $p \in s(\Sigma)$ is of type (I), (II) or (III) if it lies on a component of $\partial P$ which is of type (I), (II) or (III), respectively. 
It is of type (IV) or (V), if it lies in the interior of $P$ and has a neighborhood as shown in figures $5(\mathrm{a})$ and (b) respectively, possibly with orientations of both segments of $\partial D_{i} \cap P$ reversed for one or both $i$, where the horizontal disk is a subdisk of $P$ and the other two disks $D_{i}$ are in $D$.

Lemma 3.7. (The Unique Extension Lemma) Let $\Sigma=Q \cup P \cup D$ be a tangle complex with sink marks specified on $\partial D$, such that each $p \in s(\Sigma)$ is of one of the types (I)-(V). Then these sink marks can be uniquely extended over $\partial P$ to a branched surface structure on $\Sigma$. Moreover, if each branch on $P$ has a diamond edge on its boundary then $\Sigma$ is pre-laminar.

Proof. Checking the sink marks in figure 5, one can see that Condition (1) implies that the regular neighborhood of any singular point $p \in s(\Sigma)$ in the interior of $P$ is a branched surface. All other points of $s(X)$ are in $\partial P$, and condition (2) and Lemma 3.4 shows that there is a unique choice of sink marks for the branch curves in $\partial P$ to make a neighborhood of $\partial P$ a branched surface.

By assumption $P$ contains no sink disk because each branch $B$ has a diamond edge, which is a source edge for $B$. Since each component of $\partial P$ is of type (I), (II) or (III), no component of $D \cap Q$ is a diamond edge. Since each component $D_{i}$ of $D$ has some boundary edge on $Q$, which is a source edge for $D_{i}$, it follows that there is no sink disk on $D$. By definition each component $Q_{i}$ of $Q \mid P$ intersects $D$ at exactly one edge $\alpha$, hence the branch on $Q_{i}$ incidents $\alpha$ twice, so it has a source edge. It follows that $\Sigma$ is sinkless.

A trivial bubble $R$ is the union of some branches. Clearly $P \cup Q$ contains no sphere, so $R$ must contain a component $D_{i}$ of $D$. By definition $D_{i}$ has a boundary arc $\alpha$ on some component $Q_{j}$ of $Q$ cut along $\partial P$. Since $Q_{j}$ cut along $\alpha$ is a branch, $R$ contains $D_{i} \cup Q_{j}$, contradicting the assumption that $R$ is a sphere. Hence $\Sigma$ contains no trivial bubble.

Suppose $S$ is a closed surface carried by $\Sigma$. If $S$ intersects a fiber of a branch on $P$ then it must flow out of the diamond edge of this branch into some branch $F_{i}$ on $D$, and since $F_{i}$ has a source edge on $Q$ it must flow into $Q$. Let $m$ be a meridian loop of $Q$. Then its preimage under the collapsing map $\varphi: N(\Sigma) \rightarrow \Sigma$ is an annulus $A$ which is $I$-fibered, and $\partial A=\alpha \cup \beta$, where $\alpha$ is an arc on a single $I$-fiber, and $\beta$ is the union of a circle and an arc transverse to the $I$-fibers. If $S$ is not in $N(Q)$ then by the above it must flow into $Q$, so it intersects the $I$-fiber $\alpha$ for some meridian loop $m$. Since $S$ is transverse to the $I$-fibers, each component of $S \cap A$ is a curve in $A$ with a single boundary point on $\alpha$, hence $S \cap A$ is not a compact curve, contradicting the assumption that $S$ is a compact surface. Therefore any 
connected closed surface $S$ carried by $\Sigma$ must be disjoint from fibers of $P$ and $D$, so it is carried by $Q$ and hence is a torus carried by a component $Q^{\prime}$ of $Q$. Note that no component of $\partial P$ on $Q^{\prime}$ can be marked by diamond as otherwise it would be a source edge of a branch of $Q^{\prime}$ and hence $S$ would flow out of $Q^{\prime}$ into $P$, contradicting the fact that it is carried by $Q^{\prime}$. Therefore the inside side of $\partial N\left(Q^{\prime}\right)$ is a torus $T$ on $\partial N(\Sigma)$ with no cusp and hence is a component of $\partial_{h}(\Sigma)$, and $S$ is parallel to $T$. It follows that $\Sigma$ is atoroidal.

\section{The Hatcher-Thurston branched surfaces}

Consider $S^{3}$ as $S^{2} \times[-\infty, \infty]$ with each $S^{2} \times\{ \pm \infty\}$ pinched to a point. Denote by $S[x]$ the image of $S^{2} \times\{x\}$ and by $S[a, b]$ the image of $S^{2} \times[a, b]$.

Let $X$ be the tangle complex in $S[a, b]$ ( $a, b$ finite) with surface decomposition $(Q, P, D)$ shown in figure $6(\mathrm{a})$, where $Q$ consists of four vertical tubes represented in the figure by four vertical arcs $K, P$ is a horizontal punctured sphere, and $D$ the union of four rectangles, each having two edges on $Q$, one edge on $P$ and one edge on $\partial S[a, b]$. The four edges of $D \cap P$ are diamond edges. Fix an orientation of $K$ (so the two segments of any component of $K$ are oriented consistently), which induces sink marks on $\partial D \cap Q$. Then we see that the neighborhood of any component of $\partial P$ is a complex of type (I) as in figure 4(I). Therefore by Lemma 3.7 these sink marks extend to a unique branched surface structure on $\Sigma=Q \cup P \cup D$, which is pre-laminar.

Given two rational numbers $r_{i}=p_{i} / q_{i}$, denote by $\Delta\left(p_{1} / q_{1}, p_{2} / q_{2}\right)=$ $\left|p_{1} q_{2}-p_{2} q_{1}\right|$. When $\Delta\left(r_{1}, r_{2}\right)=1$, we can deform the branched surface above by twisting the four components of $K$ around each other so that the two top edges on $S[0]$ have slope $r_{1}$ and the bottom edges have slope $r_{2}$. (See [7] for definition of slopes of such curves.) We will call this branched surface the Hatcher-Thurston branched surface associated to the edge $e$ from $r_{1}$ to $r_{2}$, denoted by $\Sigma(e)$ or $\Sigma\left(r_{1}, r_{2}\right)$. This branched surface has the following

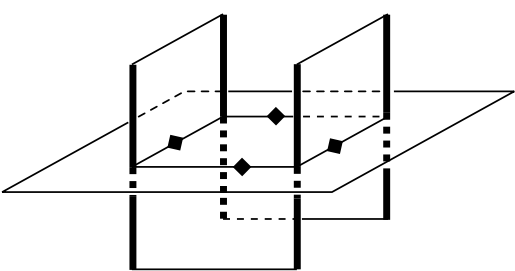

(a)

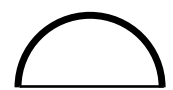

(b)

Figure 6 


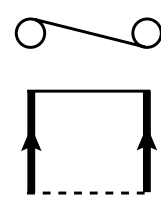

(a)

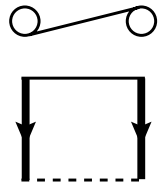

(b)

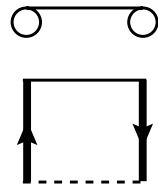

(c)

Figure 7

properties. (i) When viewed from above, we see a pair of top edges with slope $r_{1}$ on $S[a]$, and a pair of cusps on $P$ with slope $r_{2}$; (ii) when viewed from below, we see a pair of bottom edges with slope $r_{2}$ and a pair of cusps with slope $r_{1}$.

If $K$ is an oriented curve in a compact 3-manifold $M$, then a point of $K$ on $\partial M$ is positive if the orientation of $K$ points outward at that point, and negative otherwise. The boundary train tracks of $\Sigma(e)$ and the branched surfaces to be constructed below depend on the local orientations of the thick arcs $K$. These determine the sink directions and hence the way $D$ is attached to $Q$. See figures $7(\mathrm{a})-(\mathrm{c})$ for the three possible boundary train tracks when viewed from outside of $S[a, b]$, which will be said to be positively oriented, negative oriented, and antiparallel, respectively.

We refer the readers to [7, figure 4] for the Hatcher-Thurston diagram $\mathcal{D}$, which is a graph on a disk $D^{2}$ having $\mathbb{Q} \cup\{\infty\} \subset \partial D^{2}$ as vertices, with an edge connecting $r_{1}$ to $r_{2}$ if $\Delta\left(r_{1}, r_{2}\right)=1$. A path on $\mathcal{D}$ is minimal if two successive edges do not lie on a triangle of $\mathcal{D}$. Let $\mathcal{D}(p / q)$ be the subdiagram of $\mathcal{D}$ consisting of the edges of all minimal paths from $1 / 0$ to $p / q$. See figure 8 for $\mathcal{D}(3 / 11)$. When $q \neq 1 \mathcal{D}(p / q)$ is a graph on a disk $D$ containing $\partial D$, with all vertices on $\partial D$ and all faces triangles. The edges on $\partial D$ form two paths from $1 / 0$ to $p / q$, called the upper boundary path and the lower boundary path, with the upper one containing the vertices with label $r_{i}>p / q$. Edges on $\partial D$ are boundary edges, the others are interior edges. A vertex of valance at least 4 will be called a fork vertex.

If $\gamma$ is a path on $\mathcal{D}(p / q), v$ an interior vertex of $\gamma$ and $t$ the number of triangles between the two edges of $\gamma$ incident to $v$, then the corner number of $v$ on $\gamma$ is defined as $c(\gamma, v)=t$ if the triangles are above $\gamma$, and $c(\gamma, v)=-t$ otherwise. Thus any minimal path $\gamma$ from $1 / 0$ to $p / q$ can be written as $\gamma\left(c_{1}, \ldots, c_{n}\right)$, where $c_{i}=c\left(\gamma, v_{i}\right)$ and $v_{i}$ is the $i$ th vertex in the interior of $\gamma$. Denote by $\left[c_{1}, \ldots, c_{k}\right]$ the partial fraction decomposition $1 /\left(c_{1}-1 /\left(c_{2}-\ldots-1 / c_{k}\right) \ldots\right)$. Then the rational number at the vertex $v_{i}$ above is $p_{i} / q_{i}=v_{1}+\left[c_{1}, \ldots, c_{i}\right]$. In particular, $p / q=v_{1}+\left[c_{1}, \ldots, c_{n}\right]$. 


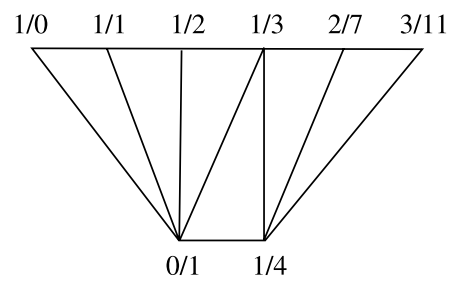

Figure 8

Note that $v_{1}$ is determined by $c_{1}$ and $p / q$ : if $m$ is the integer such that $m<p / q<m+1$ then $v_{1}=m$ if $c_{1}>0$, and $v_{1}=m+1$ if $c_{1}<0$.

Denote by $T=T_{p / q}$ the $p / q$ rational tangle in $S[-\infty, 0]$. It is the union of four vertical arcs in $S[-n, 0]$ and two arcs of slope $p / q$ on $S[-n]$ connecting the four endpoints of the vertical arcs on $S[-n]$.

Fix an orientation of $T$. Let $\gamma$ be a path in $\mathcal{D}$ from $1 / 0$ to $p / q$ with edges $e_{1}, \ldots, e_{n}$. Let $\Sigma\left(e_{i}\right)$ be the branch complex associated to the edge $e_{i}$ in $S[-i,-i+1]$, as defined above. Since the ending point of $e_{i}$ is the initial point of $e_{i+1}$ and the orientations of the thick arcs are induced by that of $T$ and hence match each other, the bottom train tracks of $\Sigma\left(e_{i}\right)$ on $S[-i]$ matches the top train tracks of $\Sigma\left(e_{i+1}\right)$, so $\Sigma(\gamma)=\Sigma\left(e_{1}\right) \cup \ldots \cup \Sigma\left(e_{n}\right)$ is a branched surface with $\alpha=\Sigma(\gamma) \cap S[0]$ a pair of train tracks of slope $1 / 0$ and $\beta=\Sigma(\gamma) \cap S[-n]$ of slope $p / q$. The bottom train tracks are antiparallel as in figure 7 (c), so it can be capped off by two copies of the trivial caps in figure 6(b). We thus obtain a branched surface $\Sigma(\gamma)$ in the complement of $T_{p / q}$.

Let $K=K_{p / q}$ be the $p / q$ 2-bridge knot or link. It can be obtained from $T$ by adding two arcs of slope $1 / 0$ on $S[0]$ connecting the endpoints of $T$. When the orientation of $T$ is induced by that of $K$, the top train tracks are antiparallel, hence they can also be capped off by trivial caps to obtain a branched surface $\hat{\Sigma}(\gamma)$ in the exterior of $K_{p / q} . \Sigma(\gamma)$ and $\hat{\Sigma}(\gamma)$ are very similar to the branched surfaces constructed in [7] and will be called the HatcherThurston branched surfaces for $T_{p / q}$ and $K_{p / q}$, respectively. By Lemma 3.7 these branched surfaces are pre-laminar. It can be shown that $\hat{\Sigma}(\gamma)$ is also laminar if $\gamma$ is minimal, see the proof of Theorem 5.3.

\section{Delman channels and channel surfaces}

A laminar branched surface $\Sigma$ in the exterior of a knot $K$ is persistently laminar if it remains laminar in $K(r)$ for all non-meridional slopes $r$. To create a persistently laminar surface we need to modify the construction of 


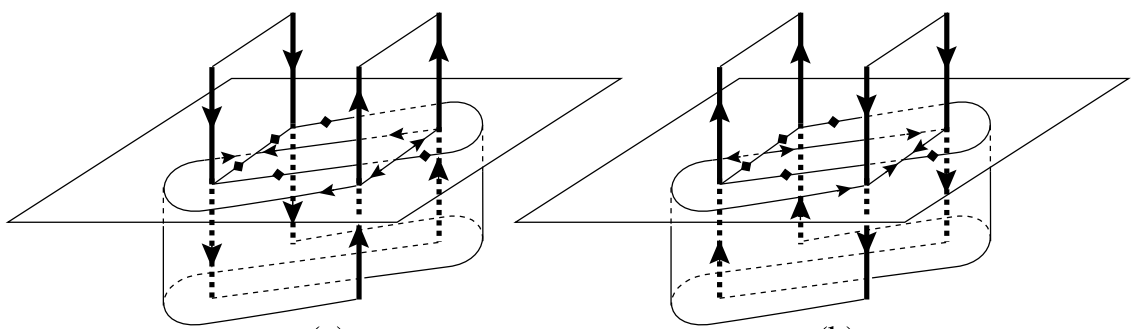

(a)

(b)

Figure 9

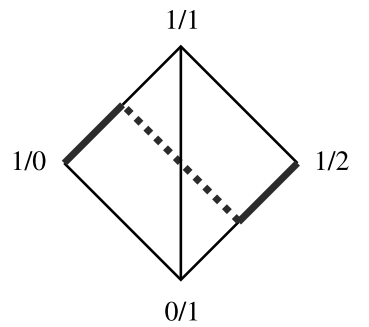

(a)

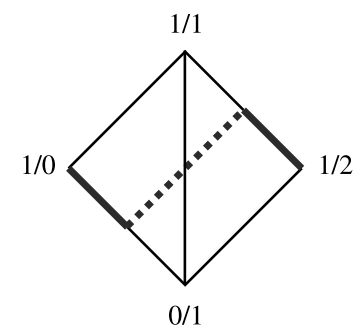

(b)

Figure 10

the Hatcher-Thurston branched surface to create some meridional cusps. The following is a construction of Delman's channel branched surface [4].

Let $\Sigma=Q \cup P \cup D$ be the 2-complex in $S[a, b]$ shown in figure 9(a), where $Q$ consists of four vertical tubes represented by the four arcs $K$ in the figure, $P$ is a horizontal sphere, and $D$ is the union of four disks of type $\alpha \times I$, where $\alpha$ is an arc of slope $1 / 0$ for the two disks $D_{1}, D_{2}$ above $P$, and of slope $1 / 2$ for the two disks $D_{3}, D_{4}$ below $P$. The sink marks are shown in figure 9(a). Let $\alpha_{i}=D_{i} \cap P$. Unlike the Hatcher-Thurston complex, only two of these $\operatorname{arcs} \alpha_{1}, \alpha_{3}$ are diamond edges, the other two are marked with orientation arrows. Note that the orientation of $\alpha_{2}, \alpha_{4}$ changes when passing across a diamond edge, hence the two singular points in the interior of $P$ are of type (IV) as in Definition 3.6. The two components of $\partial P$ on the left are of type (I) and the two on the right are of type (II), therefore by Lemma 3.7 these sink marks extend to a unique branched surface structure on $\Sigma$. The two components of $\partial P$ of type (II) produce two meridional cusps.

By definition the sink direction points to the right of an oriented arc of $\partial D_{i}$ when standing on $P$ on the side $D_{i}$ is attached and facing to the 
direction of the orientation mark. The cusp of that arc is then on the left. When passing across a diamond edge the cusp continues onto the diamond edge on the side of the cusp. Using this fact one can check that the cusps on the top side of $P$ in figure $9(\mathrm{a})$ are of slope $1 / 1$, while those on the bottom side of $P$ are of slope $0 / 1$.

On the Hatcher-Thurston diagram $\mathcal{D}$, the branched surface in figure 9 (a) is represented by a Delman channel of type $A$ shown in figure 10(a), which is an arc starting from the vertex $1 / 0$, going half way towards the vertex $1 / 1$, then jump to the middle of the edge from $0 / 1$ to $1 / 2$ and finish at the vertex $1 / 2$. It reflects the properties of $\Sigma$ that the top train tracks have slope $1 / 0$, the cusps on the top side of $P$ are of slope $1 / 1$, the cusps on the bottom side of $P$ are of slope $0 / 1$ and the bottom train tracks have slope $1 / 2$.

We may reverse the orientations of all the edges in figure 9 (a) to obtain the one in figure $9(\mathrm{~b})$. As above, these sink marks determines a branched surface structure on the 2-complex. By checking the cusps one can show that the top cusps are of slope $0 / 1$ while the bottom ones are of slopes $1 / 1$. Therefore it corresponds to the path in figure 10(b), called a Delman channel of type $B$.

The branched surfaces in figure 9 are called the Delman channel surfaces, denoted by $\Sigma(e)$ if $e$ is the corresponding Delman channel. It is of type A or type $\mathrm{B}$ according to the type of $e$. Note that each boundary arc of $\Sigma(e)$ must connect a pair of parallel endpoints of the $\operatorname{arcs} K$ representing $Q$, so that the corresponding boundary train tracks are one positive and one negative, as in figures 7(a)-(b), respectively.

By twisting the four vertical arcs around, we can isotope this surface $\Sigma(e)$ in $S[a, b]$ to change the top slope to $r_{1}=p_{1} / q_{1}$ and the bottom slope to $r_{2}=p_{2} / q_{2}$ if $\Delta\left(r_{1}, r_{2}\right)=2$. Hence we can embed a Delman channel into a pair of adjacent triangles in the Hatcher-Thurston diagram $\mathcal{D}$. The only requirement is that the curves $K$ must be oriented in such a way that the two train tracks of slope $r_{1}$ on $S[a]$ connecting the four points of $K \cap S[a]$ must be one positive and one negative, as in figures $7(\mathrm{a})$ and (b), respectively. One can see that the two train tracks of slope $r_{2}$ on $S[b]$ also have the same property.

Now consider $L=T_{p / q}$ or $K_{p / q}$. It intersects the level sphere $S[0]$ in four points. Recall that two points of $L$ on a level sphere are parallel if the orientations of $L$ at these two points are both upward or both downward. A rational number $p / q$ is assigned a parity pair $o / e, e / o$ or $o / o$ if $(p, q)$ is (odd, even), (even, odd) or (odd, odd), respectively. Note that two arcs on $S[0]$ with the same parity pair connect the same pair of points of $L \cap S[0]$, 
so we can use the slope $1 / 0,0 / 1$ or $1 / 1$ that has the same parity pair as $p / q$ to determine whether a curve of slope $p / q$ connects a pair of parallel points.

Definition 5.1. Let $L=T_{p / q}$ or $K_{p / q}$. A path $\gamma$ in $\mathcal{D}(p / q)$ from $1 / 0$ to $p / q$ is an allowable path if it satisfies the following conditions:

(1) it is the union of edges of $\mathcal{D}(p / q)$ and Delman channels;

(2) it is minimal in the sense that the corner number $c\left(\gamma, v_{i}\right)$ defined in Section 4 satisfies $\left|c\left(\gamma, v_{i}\right)\right| \geq 2$ for any interior vertex $v_{i}$ of $\gamma$;

(3) the label of the ending points of the Delman channels in $\gamma$ all have the same parity pair, which is different from that of $p / q$, and is also different from that of $1 / 0$ if $L=K_{p / q}$.

Write $\gamma=e_{1} \cup \ldots \cup e_{n}$, where each $e_{i}$ is either an edge or a Delman channel. Note that the beginning and ending points of a Delman channel $e_{i}$ have the same parity pair. Let $r_{i}^{\prime}, r_{i}^{\prime \prime}$ be the slopes of the beginning and ending points of the $i$-th Delman channel in $\gamma$. Then condition (3) above implies that these all have the same parity pair, it is different from that of $p / q$, and if $L$ is a knot then it is also different from that of $1 / 0$. This implies that we can orient $L$ so that an arc of slope $r_{i}^{\prime}$ or $r_{i}^{\prime \prime}$ connects a pair of parallel points of $L$, so there is a Delman channel surface $\Sigma\left(e_{i}\right)$ whose sink marks on $Q$ coincide with this orientation of $L$. As in the construction of the Hatcher-Thurston branched surfaces for minimal path, we can now construct branched surfaces $\Sigma(\gamma)$ and $\hat{\Sigma}(\gamma)$ for $\gamma$ as the union of $\Sigma\left(e_{i}\right) \subset S[-i,-i+1]$ and some copies of the trivial caps in figure $6(\mathrm{~b})$ at the top and bottom, except that when $e_{i}$ is a Delman channel $\Sigma\left(e_{i}\right)$ is the corresponding Delman channel surface above instead of the Hatcher-Thurston branched surface in figure 6 . The branched surfaces $\Sigma(\gamma)$ in $S[-\infty, 0]$ and $\hat{\Sigma}(\gamma)$ in $S^{3}$ are called the Delman branched surfaces for the tangle $T_{p / q}$ and the link $K_{p / q}$, respectively, corresponding to the allowable path $\gamma$.

Lemma 5.2. Let $\Sigma=\Sigma(\gamma)$ and $\hat{\Sigma}=\hat{\Sigma}(\gamma)$ be the Delman branched surfaces corresponding to an allowable path $\gamma=\gamma\left(c_{1}, \ldots, c_{n}\right)$ in $\mathcal{D}(p / q)$, where $c_{i}=$ $c\left(\gamma, v_{i}\right)$ is the corner number of $\gamma$ at $v_{i}$. Then each of $E(\hat{\Sigma})$ and $E(\Sigma)$ has $n+1$ components $Y_{0}, \ldots, Y_{n}$, one for each vertex $v_{i}$ of $\gamma$, such that $Y_{n}$ for both branched surfaces and $Y_{0}$ for $\hat{\Sigma}$ are 3-balls with a single cusp, and $Y_{i}$ is a solid torus with cusp winding number $\left|c_{i}\right|$ for $i \neq 0, n$.

Proof. Topologically the exterior of $\hat{\Sigma}(\gamma)$ is obtained by cutting $S^{3}$ along horizontal spheres $S[-i+1 / 2]$ for $i=1, \ldots, n$, then removing a regular neighborhood of the attaching disks $D$ in each region. Hence it has $n+1$ regions $Y_{0}, \ldots, Y_{n}$, one for each vertex $v_{i}$. It is easy to see that $Y_{0}$ and $Y_{n}$ are 3 -balls 
with a single cusp. For $i=1, \ldots, n-1, D \cap S[-i-1 / 2,-i+1 / 2]$ is a pair of disks $D_{i 1} \cup D_{i 2}$, each $D_{i j}$ is a $\alpha_{j} \times I$ for a curve $\alpha_{j}$ of slope $p_{i} / q_{i}$, hence $Y_{i}$ is a solid torus. The exterior of $\Sigma(\gamma)$ is the same, except that $Y_{0}$ is now a solid torus in $S[-1 / 2,0]$.

We need to determine the winding number of the cusps on $\partial Y_{i}$. By an isotopy we can deform $\hat{\Sigma}(\gamma)$ so that the curves $\alpha_{j}$ above have slope $1 / 0$, and the cusps on the bottom side of $S[-i+1 / 2]$ are of slope $0 / 1$. The deformation changes labels of all vertices of $\mathcal{D}$ but preserves $\Delta(r, s)$. Whether $e_{i}$ is an edge or Delman channel, the ending segment of $e_{i}$ now lies on the edge from $1 / 0$ to $0 / 1$, so the initial segment of $e_{i+1}$ must be on the edge from $0 / 1$ to $1 / m$ for some integer $m$ because all vertices connected to $0 / 1$ are of that form. Moreover, the corner number $c\left(\gamma, v_{i}\right)=m$. It follows that the cusps on the top of $S[-i-1 / 2]$ are of slope $1 / m$. It is now easy to see that the minimal intersection number between a meridian disk of $Y_{i}$ and the cusp on $\partial Y_{i}$ is $|m|=\left|c\left(\gamma, v_{i}\right)\right|=\left|c_{i}\right|$.

The following theorem is an extension of a result of Delman [De] for these branched surfaces, which has been used in [3] to determine small Seifert fibered surgeries on 2-bridge knots.

Theorem 5.3. Given an allowable path $\gamma$ from $1 / 0$ to $p / q$, the corresponding branched surfaces $\Sigma=\Sigma(\gamma)$ and $\hat{\Sigma}=\hat{\Sigma}(\gamma)$ are pre-laminar. $\hat{\Sigma}$ is laminar in $E\left(K_{p / q}\right)$, and is genuine if at least one vertex $v$ on $\gamma$ has $|c(\gamma, v)|>2$. If $\gamma$ has $k$ Delman channels then $\Sigma$ and $\hat{\Sigma}$ have $2 k$ meridional cusps.

Proof. The first statement follows from Lemma 5.2. If $\gamma=\gamma\left(c_{1}, \ldots, c_{n}\right)$ is an allowable path then $\left|c_{i}\right| \geq 2$ for all $i$, so by Lemmas $5.2 E(\hat{\Sigma}(\gamma))$ is an essential cusped manifold, and by Lemma $2.2 \hat{\Sigma}(\gamma)$ is a laminar branched surface. If some $\left|c_{i}\right| \geq 3$ then the corresponding region $Y_{i}$ in Lemma 5.2 is not an $I$-bundle, hence $\hat{\Sigma}(\gamma)$ is genuine. Since each Delman channel creates two meridional cusps, $\hat{\Sigma}$ and $\Sigma$ have $2 k$ meridional cusps if $\gamma$ contains $k$ Delman channels.

\section{Half channel surfaces}

We can reverse the orientations of some of the edges of the Delman channel surface in figure 10 to obtain new branched surfaces. The ones in figures $11\left(a_{1}\right)-\left(b_{3}\right)$ are called Delman half channel surfaces, and the corresponding paths shown in the figure are their Delman half channels. Some of these are constructed in [4] in a more sophisticated way. As before, denote by $Q$ the four tubes represented by the four vertical $\operatorname{arcs} K, P$ the 

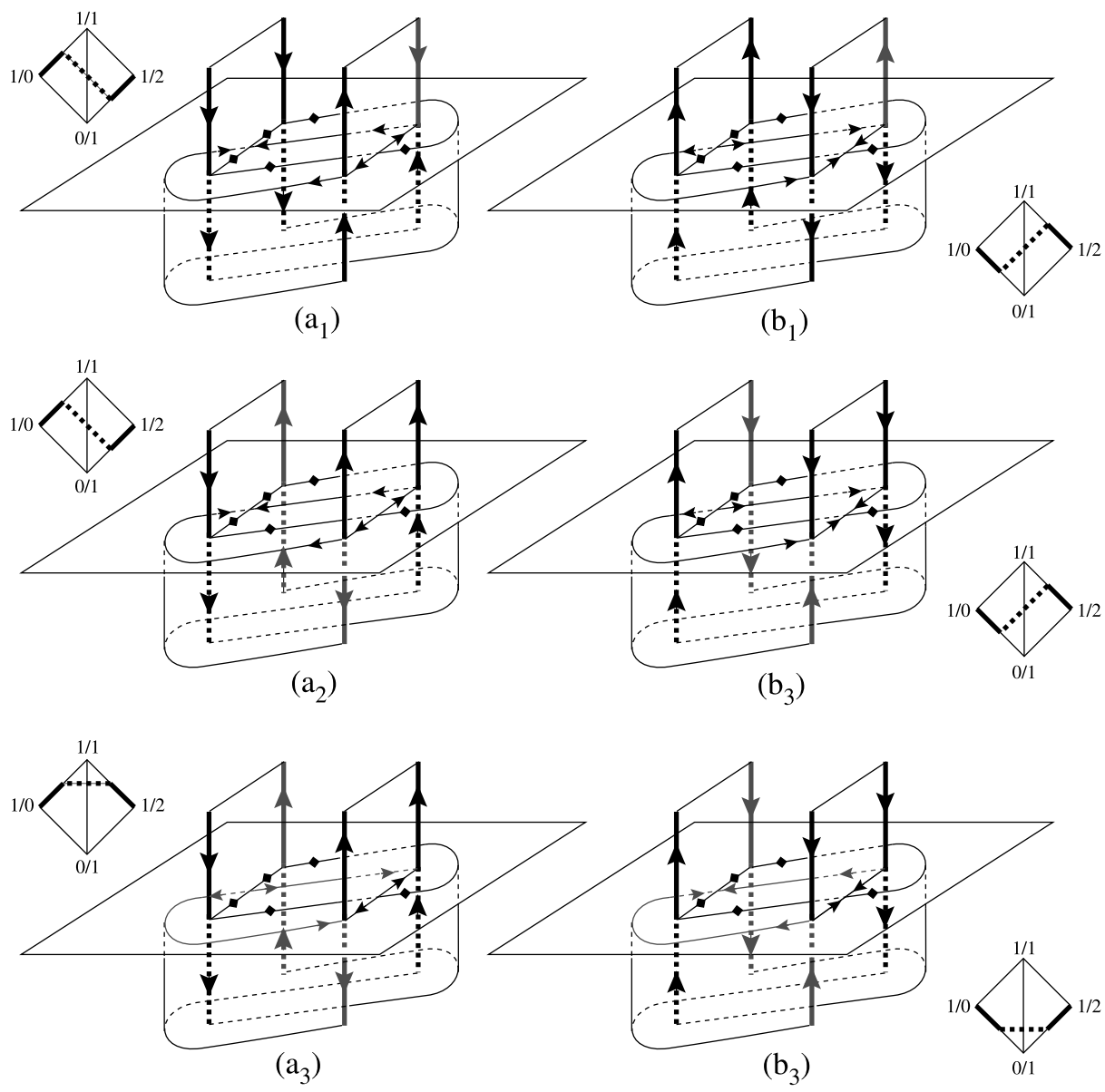

Figure 11

horizontal punctured sphere and $D$ the four disks attached to $Q \cup P$. We use $\Sigma\left(x_{i}\right)=Q \cup P \cup D$ to denote the 2-complexes with sink marks in figure $11\left(x_{i}\right), x=a, b$ and $i=1,2,3$. For $i=2,3$ we allow the orientations of both of the two left edges in $\Sigma\left(x_{i}\right)$ be changed simultaneously, so there are two choices of $\Sigma\left(x_{i}\right)$ in this case.

Lemma 6.1. (1) $\Sigma\left(x_{i}\right)$ are branched surfaces.

(2) Each $\Sigma\left(x_{i}\right)$ has one meridional cusp.

(3) If the Delman half channel $\gamma$ representing $\Sigma\left(x_{i}\right)$ has starting edge from $1 / 0$ to $r_{1}$ and ending edge from $r_{2}$ to $1 / 2$ then the cusp above $P$ has slope $r_{1}$ and the one below $P$ has slope $r_{2}$. 
Proof. As for the Delman channel surfaces, (1) and (2) can be verified using Lemma 3.7. Note that in each case $\partial P$ has two components of type I, one component of type II and one component of type III. Therefore there is exactly one meridional cusp in each case.

(3) The cusp slopes on the two sides of $P$ are determined by the diamond edges and the orientation sink marks on $\partial D \cap P$. When $i=1,2$ the sink marks on $P$ are the same as those in figure 9 , so the Delman half channel looks the same as those for the Delman channels in figure 10. In figures $11\left(a_{3}\right)-\left(b_{3}\right)$ the orientations of $\partial D \cap P$ have been reversed for one of the disks below $P$. One can verify that it changes the cusp slope below $P$ to the one indicated by the Delman half channel shown in the figure.

Let $\gamma$ be a path in $\mathcal{D}(p / q)$ consisting of edges and a single half channel $\tau$ of type $x_{i}$. Denote $\Sigma\left(x_{i}\right)$ by $\Sigma(\tau)$. We can construct a 2 -complex $\Sigma(\gamma)$ in the 3 -ball $S[-\infty, 0]$ starting with the half channel surface $\Sigma(\tau)$, then adding Hatcher-Thurston surfaces $\Sigma(e)$ successively for the edges $e$ before and after $\tau$. Recall that there are two possible choices of $\Sigma(\tau)$ if $i>1$. We say that $\gamma$ satisfies the orientation requirement if one can choose $\Sigma(\tau)$ so that the two bottom train tracks of $\Sigma(\tau) \cup\left(\cup_{e} \Sigma(e)\right)$ can be capped off by trivial caps in figure 6 (b) to form a branched surface, which will be denoted by $\Sigma(\gamma)$.

We say that $\gamma$ has starting slope $r$ if the initial segment of $\gamma$ is on the edge from $1 / 0$ to $r$.

Lemma 6.2. Suppose $\gamma$ is a path in $\mathcal{D}(p / q)$ from $1 / 0$ to $p / q$, containing a half channel $\tau$ of type $x_{i}$. Then $\gamma$ satisfies the orientation requirement unless $i=1$ and the initial point (and hence the ending point) of $\tau$ has the same parity pair as that of $p / q$.

Proof. Let $\Sigma(\tau)$ be a branched surface in $S[a, b]$ corresponding to the half channel $\tau$ of type $x_{i}$. When $i>1$ we have two choices of $\Sigma(\tau)$ and the second one is obtained from that in figure $11\left(x_{i}\right)$ by reversing the orientations of the two arcs on the left, hence one of them has the property that an arc of slope $p / q$ on the bottom level sphere $S[a]$ connect a pair of antiparallel endpoints of the four vertical arcs $K$ in figure $11\left(x_{i}\right)$. The bottom train track of $\Sigma(\tau) \cup\left(\cup_{e} \Sigma(e)\right)$ has slope $p / q$, therefore the above implies that these train tracks are antiparallel as in figure $7(\mathrm{c})$; hence one can cap it off using the trivial caps to obtain a branched surface $\Sigma(\gamma)$.

Now assume $i=1$. In this case the train tracks at the bottom of $\Sigma(\gamma)$ are one positive and one negative, as in figures $7(\mathrm{a})-(\mathrm{b})$. If the endpoint of $\tau$ has different parity pair from that of $p / q$ then an arc of slope $p / q$ connects a pair of antiparallel edge endpoints of $K$ (with piecewise orientation induced 
by sink marks), hence $\Sigma(\tau) \cup\left(\cup_{e} \Sigma(e)\right)$ can be capped off by trivial caps to make a branched surface.

Definition 6.3. A path $\gamma$ in $\mathcal{D}(p / q)$ from $1 / 0$ to $p / q$ consisting of edges and one half channel is a semi-allowable path if (1) it satisfies the orientation requirement above, and (2) it is minimal in the sense that the corner number $c\left(v_{i}, \gamma\right)$ defined in Section 4 satisfies $\left|c\left(v_{i}, \gamma\right)\right| \geq 2$ for any interior vertex $v_{i}$ of $\gamma$. The path $\gamma$ is genuine if $\left|c\left(v_{i}, \gamma\right)\right| \geq 3$ for some $i$.

Lemma 6.4. Let $\gamma$ be a semi-allowable path in $\mathcal{D}(p / q)$ with starting slope $r$ and corner number $c_{i}$ at the ith vertex. Then the exterior of $\Sigma(\gamma)$ has $n+1$ components $Y_{0}, \ldots, Y_{n}$, one for each vertex $v_{i}$ of $\gamma$, such that $Y_{n}$ is a 3-ball with a single cusp, and $Y_{i}$ is a solid torus with cusp winding number $\left|c_{i}\right|$ for $i \neq 0, n$. The cusps above the top level surface is of slope $r$.

Proof. This is similar to Lemma 5.2. We omit the details.

Note that if $\gamma$ contains a half channel and if $T_{p / q}$ is endowed with the induced piecewise orientation, then exactly three of the four endpoints of $T_{p / q}$ on the top level sphere $S[0]$ have the same orientation. We say that $\gamma$ is upward if the orientations at those three points are upward, and downward otherwise. From figure 11 we see that $\gamma$ is upward if and only if the half channel in it is of type $b_{1}, a_{2}$ or $a_{3}$.

Proposition 6.5. Suppose $0<p / q<1$. Let $\mathcal{D}=\mathcal{D}(p / q)$.

(1) $\mathcal{D}$ always has an upward semi-allowable path $\gamma$ with starting slope 1 , and there is a genuine such $\gamma$ unless $p=1$ or $q-1$.

(2) $\mathcal{D}$ has a downward semi-allowable path $\gamma$ with starting slope 1 unless $p=1$, or $p=q-1$ and $q$ is even. $\mathcal{D}$ has a genuine such $\gamma$ unless $p=1,2$ or $q-1$.

(3) $\mathcal{D}$ always has a downward semi-allowable path $\gamma$ with starting slope 0 , and there is a genuine such $\gamma$ unless $p=1$ or $q-1$.

(4) $\mathcal{D}$ has an upward semi-allowable path $\gamma$ with starting slope 0 unless $p=q-1$, or $p=1$ and $q$ is even. $\mathcal{D}$ has a genuine such $\gamma$ unless $p=1, q-2$ or $q-1$.

Proof. (1) If $p / q \geq 1 / 2$, let $\gamma$ be the half channel of type $a_{2}$ in figure $11\left(a_{2}\right)$ followed by an edge path on the lower boundary of $\mathcal{D}$; if $p / q<1 / 2$, let $\gamma$ be the half channel of type $a_{3}$ followed by a path on the upper boundary of $\mathcal{D}$. By Lemma $6.2 \gamma$ satisfies the orientation requirement and hence is semi-allowable. If $p \neq 1, q-1$ then there are fork vertices on both the upper and lower boundary of $\mathcal{D}$, so $|c(v)|>2$ for some $v \in \gamma$, hence $\gamma$ is genuine. 


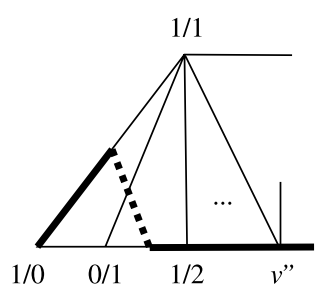

(a)

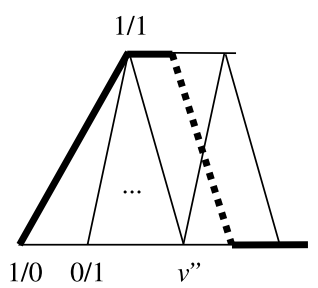

(b)

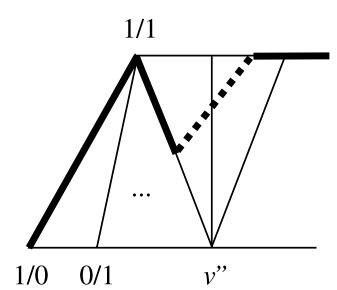

(c)

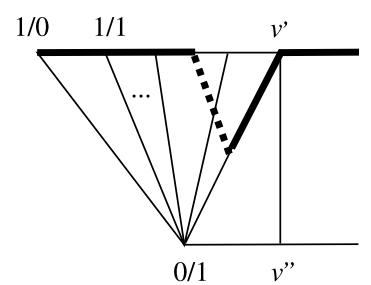

(d)

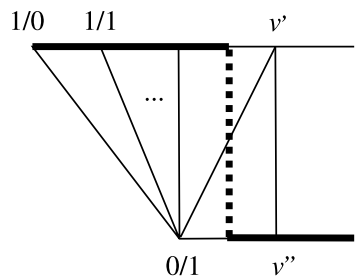

(e)

Figure 12

(2) First assume $p / q>1 / 2$. Then the vertex $1 / 2$ is on the lower boundary of $\mathcal{D}$. If $q$ is odd then we may choose $\gamma$ to be the half channel in figure $11\left(a_{1}\right)$ followed by an edge path on the lower boundary of $\mathcal{D}$, as shown in figure $12(\mathrm{a})$. By Lemma 6.2 this is semi-allowable since $1 / 2$ has different parity pair from that of $p / q$, and it is genuine if $p \neq q-1$ because in this case there is at least one fork vertex at the lower boundary.

If $q$ is even, then $p \neq q-1$ by assumption, so the last interior edge at $v^{\prime}=1 / 1$ connects it to a fork vertex $v^{\prime \prime}$ on the lower boundary. Let $\gamma$ be the path in figures $12(\mathrm{~b})-(\mathrm{c})$ according to whether $v^{\prime \prime}$ has only two interior edges or more, where the half channel is of type $a_{1}$. Since $1 / 1$ has parity pair different from that of $p / q$, by Lemma $6.2 \gamma$ is semi-allowable. It is genuine unless $\left|c\left(v^{\prime}, \gamma\right)\right|=2$ (so $\gamma$ is as shown in figure $6.2(\mathrm{c})$ ), and there is no fork vertex on the upper boundary other than $1 / 1$. However, in this case $v^{\prime \prime}=1 / 2$, and it has an edge connected to $p / q$, so $q$ cannot be even, a contradiction. Therefore $\gamma$ is genuine.

Now assume $p / q<1 / 2$, so the vertex $1 / 2$ is on the upper boundary of $\mathcal{D}$. Let $v^{\prime}$ be the first fork vertex on the upper boundary, which exists since $p \neq 1$. Let $\gamma_{1}$ and $\gamma_{2}$ be the paths in figures $12(\mathrm{~d})-(\mathrm{e})$, respectively. Each $\gamma_{i}$ is the union of a single half channel $\tau$ of type $a_{1}$ and some boundary edges. 
It is semi-allowable unless the ending point of $\tau_{i}$ has the same parity pair as that of $p / q$. Since the ending points of $\tau_{1}$ and $\tau_{2}$ are connected by an edge of $\mathcal{D}$, they have different parity pair. It follows that at least one of the $\gamma_{i}$ is semi-allowable. It remains to show that $\gamma_{i}$ can be chosen to be genuine if $p \neq 1,2, q-1$.

If $\gamma_{1}$ is semi-allowable but not genuine, then $v^{\prime}$ is the only fork vertex on the upper boundary, and it has only two interior edges, so $v^{\prime}, p / q$ and all the vertices between them on the upper boundary connect to the same vertex on the lower boundary, which must be the vertex $v^{\prime \prime}$ in figure 12(d) because there is no edge connecting $v^{\prime}$ to any vertex between $v^{\prime \prime}$ and $p / q$ on the lower boundary. Therefore $v^{\prime \prime}$ and $p / q$ are connected, so they have different parity pairs, which implies that $\gamma_{2}$ is also semi-allowable. Hence either $\left|c\left(v^{\prime \prime}, \gamma_{2}\right)\right| \geq 3$ and we are done, or there are no vertices between $v^{\prime}$ and $p / q$, in which case $p / q=1 /(a+1 / 2)=2 /(2 a+1)$, hence $p=2$ and the result follows.

If $\gamma_{1}$ is not semi-allowable then $\gamma_{2}$ is semi-allowable, so if $\gamma_{2}$ is not genuine then all vertices between $v^{\prime \prime}$ and $p / q$ (including the two endpoints) would have edge connected to $v^{\prime}$, which implies $v^{\prime}$ is connected to $p / q$ and hence has different parity pair from that of $p / q$, so $\gamma_{1}$ is also semi-allowable, which is a contradiction.

The proofs of (3) and (4) are similar, using the half channels of types $b_{i}$ instead.

Theorem 6.6. Let $K$ be a non-2-bridge Montesinos knot. Then $K$ has a persistently laminar branched surface in its complement unless it is equivalent to $K\left(1 / q_{1}, 1 / q_{2}, 1 / q_{3},-1\right)$, where $q_{i}$ are positive integers, and $q_{1}$ is even.

Proof. If the length of $K$ is at least 4 then by [10] the exterior of $K$ contains a closed essential surface which remains incompressible after all surgery on $K$. Hence we may assume that $K$ is of length 3 . Write $K=K\left(r_{1}, r_{2}, r_{3}, n\right)$, where $0<r_{i}=p_{i} / q_{i}<1$ and $n$ is an integer. By taking the mirror image if necessary we may assume that $n \geq-1$. We may assume that $q_{2}, q_{3}$ are odd.

First assume $n \geq 0$. By Proposition 6.5(1) there is an upward semiallowable path $\gamma_{1}$ in $\mathcal{D}\left(r_{1}\right)$ with starting slope 1 , and there is a downward semi-allowable path $\gamma_{2}$ in $\mathcal{D}\left(r_{2}\right)$ with starting slope 0 . Let $\gamma_{3}$ be the upper boundary path in $\mathcal{D}\left(r_{3}\right)$, which has starting slope 1 . Let $\Sigma\left(\gamma_{i}\right)$ be the branched surface constructed above for $i=1,2$, and let $\Sigma\left(\gamma_{3}\right)$ be the Hatcher-Thurston branched surface, with the arcs of $T\left(r_{3}\right)$ oriented so that the two left edges points outward. (This is possible since $q_{3}$ is odd.) Inserting 


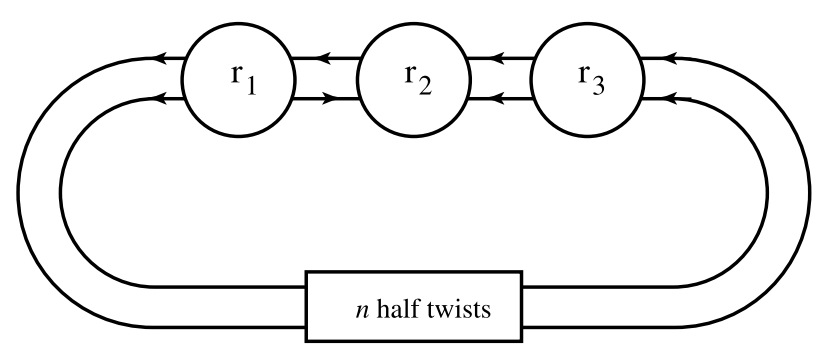

Figure 13

these into a band with $n$ half twist produces a branched surface $\Sigma$ in the knot exterior as shown in figure 13. By Lemma $3.7 \Sigma$ is pre-laminar. Since $\gamma_{1}$ and $\gamma_{3}$ has starting slope 1 and $\gamma_{2}$ has starting slope 0 , the component of $E(\Sigma)$ outside of the tangles is the complement of a band with $n+2$ half twists, and it has the boundary of the band as its cusps, so it is an essential cusped manifold. Any other component of $E(\Sigma)$ is a component of the exterior of some $\Sigma\left(\gamma_{i}\right)$ in the corresponding tangle space, which by Lemma 6.4 is a solid torus with cusp winding number at least 2 . Hence $\Sigma$ is laminar by Lemma 2.2. Since it has two meridional cusps, it remains laminar after all Dehn surgery.

Now assume $n=-1$. If for some $i, q_{i}$ is odd and $p_{i} \neq 1$, or $q_{i}$ is even and $p_{i} \neq 1, q_{i}-1$, then by Proposition 6.5(2) we have a downward semi-allowable path $\gamma_{i}$ with starting slope 1 . We can then choose $\gamma_{j}(j=1$ if $i \neq 1)$ to be an upward semi-allowable path with starting slope 1 and $\gamma_{k}(k \neq i, j)$ the upper boundary path, and construct $\Sigma$ as above. Since each of these paths has starting slope 1 , the outside component of $\Sigma$ has $n+3=2$ half twists and hence for the same reason as above, $\Sigma$ is a persistently laminar branched surface.

If $q_{1}$ is also odd then the above shows that $p_{i}=1$ for all $i$, in which case $K\left(1 / p_{1}, 1 / p_{2}, 1 / p_{3},-1\right)$ is a link of two components, contradicting the assumption. Therefore we may assume $q_{1}$ is even. By the above it remains to consider the case that $p_{1}=q_{1}-1$, and $p_{2}=p_{3}=1$. If $q_{1}=2$ then $p_{1}=1$ and we are done, so we may assume $q_{1} \geq 4$. Here the construction is different. In this case $\gamma_{1}$ is a path with two half channels, as shown in figure 14(a). The half channel at the end is of type $b_{2}$. The one at the beginning is a new half channel with its corresponding branched surface shown in figure 14(b), which is obtained from the one in figure $11\left(a_{2}\right)$ by rotating upside down along the horizontal axis from left to right, and then twisting the strings so that the top train track has slope $1 / 0$. One can also check directly from figure 14(b) that it is a branched surface with a single meridional cusp, the 


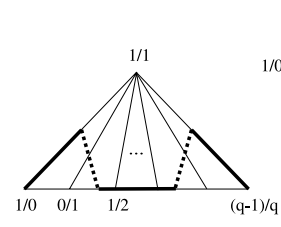

(a)

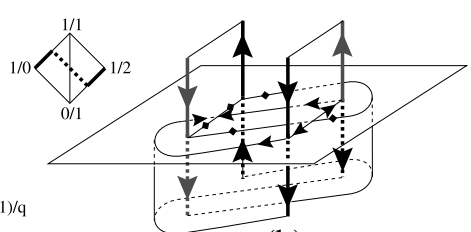

(b)

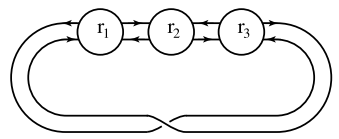

(c)

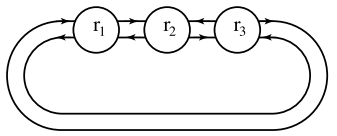

(d)

Figure 14

cusp on the top side of $P$ has slope 1 and the one on the bottom side of $P$ has slope 0 . Now the orientations of the arcs at the bottom level of this surface match the orientation of the top arcs of one of the surfaces of type $b_{2}$ (the one with orientations of both left arcs of that in figure $11\left(b_{2}\right)$ reversed). We can then add some Hatcher-Thurston surfaces corresponding to the edges between these two half channels if necessary to obtain a branched surface $\Sigma\left(\gamma_{1}\right)$ corresponding to the path $\gamma_{1}$ of $\mathcal{D}\left(r_{1}\right)$ in figure $14(\mathrm{a})$. The other two tangles are of type $T_{1 / q_{i}}$ with $q_{i}$ odd, so we can orient them as shown in figure $14(\mathrm{c})$. Let $\gamma_{i}$ be the upper boundary path in $\mathcal{D}\left(1 / q_{i}\right)$, which has starting slope 1 , and let $\Sigma\left(\gamma_{i}\right)$ be the corresponding Hatcher-Thurston branched surface. Inserting these into the band with $n=-1$ half twist as in figure $14(\mathrm{c})$, we obtain the branched surface $\Sigma$. For the same reason as before, $\Sigma$ is a persistently laminar branched surface.

We note that in the construction above we may reverse the orientations of both left edges in the half channel surfaces in figure 14(b) and figure $11\left(b_{2}\right)$, so the outside orientations of $\Sigma\left(\gamma_{1}\right)$ is obtained from that in figure 14(c) by reversing the orientations of the two arcs on the left of the tangle, as shown in figure $14(\mathrm{~d})$. The orientations would not match that of the right endpoints of the third tangle, but they do when there is no half twist or an even number of half twists at the bottom, as shown in the figure for $n=0$. Therefore this modification produces a persistently laminar branched surface $\Sigma$ when $n \geq 0$ is even. This modification is needed in the proof of Theorem 6.7.

Theorem 6.7. Let $K$ be a Montesinos knot of length 3. Then $K$ has a genuine persistently laminar branched surface in its complement unless $K$ is equivalent to $K\left(1 / q_{1}, 1 / q_{2}, p_{3} / q_{3}, n\right)$, such that either 
(1) $n=0, q_{i} \geq 2$, and $p_{3}=1$; or

(2) $n=-1, q_{i} \geq 2$, and $p_{3}=1,2$ or $q_{3}-1$.

Proof. As before, we may assume that $K=K\left(r_{1}, r_{2}, r_{3}, n\right)$, where $0<r_{i}=$ $p_{i} / q_{i}<1$ and $n \geq-1$. If $n>0$, or if $n=0$ and some $p_{i} \neq 1$ then we can construct $\gamma_{i}$ and $\Sigma$ as in the proof of Theorem 6.6 for the case $n=-1$. Now the outside component of the exterior of $\Sigma$ is the exterior of a band with at least three half twists, hence $\Sigma$ is genuine. (Unlike Theorem 6.6, we cannot claim that $q_{1}$ is even in this case, because the proof of Theorem 6.6 used the fact that $K\left(1 / q_{1}, 1 / q_{2}, 1 / q_{3}, n\right)$ is a link of two components if all $q_{i}$ are odd and $n=-1$, which is no longer true when $n=0$.)

We may now assume that $n=-1$. The result is true if $p_{1}=p_{2}=p_{3}=1$, or if $p_{1}=p_{2}=1$ and $p_{3}=q_{3}-1$. Thus we may assume that $p_{3} \neq 1$, and either $p_{1} \neq 1$ or $p_{3} \neq q_{3}-1$. Note that if $p_{1} \neq 1$ then up to relabeling of the tangles we may assume that $q_{3}$ is odd. Hence either $p_{3} \neq q_{3}-1$ or $q_{3}$ is odd. We may now apply Proposition 6.5(2) to obtain a downward semi-allowable path $\gamma_{3}$ in $\mathcal{D}\left(p_{3} / q_{3}\right)$ with starting slope 1 . If $p_{j} \neq 1$ for some $j=1,2$, let $\gamma_{j}$ be the upper boundary path in $\mathcal{D}\left(p_{j} / q_{j}\right)$, which is genuine in this case, and let the other $\gamma_{k}$ be an upward semi-allowable path with starting slope 1 , as in Proposition 6.5(1). If $p_{1}=p_{2}=1$ but $p_{3} \neq 1,2, q_{3}-1$ then by Proposition $6.5(2)$ we may choose $\gamma_{3}$ to be genuine. It follows that the branched surface $\Sigma$ corresponding to these paths is genuine unless $p_{1}=p_{2}=1$ and $p_{3}=1,2$ or $q_{3}-1$.

Example 6.8. The knot $10_{142}$ on the knot table of [9] is the pretzel knot $K(1 / 3,1 / 3,-1 / 4)$. It is one of the five knots in Gabai's Frontier Question FQ 1.2 [5] that were not known whether all surgeries are laminar. Theorem 6.6 shows that it is persistently laminar.

The construction of branched surfaces in rational tangle spaces can be used to construct persistently laminar branched surfaces in the complement of some non-Montesinos knots. Here is an example. Let $L$ be a non-split oriented link. A spanning surface $F$ of $L$ is $\pi_{1}$-injective if it is $\pi_{1}$-injective in the complement of $L$, in which case the manifold $M$ obtained by cutting $E(L)$ along $F$ is an essential cusped manifold with $\partial F$ as its cusp. Let $\alpha$ be a proper arc in $F$. Embed a regular neighborhood $D$ of $\alpha$ in $\mathbb{R}^{2}$ so that the two arcs $L \cap \partial D=a_{1} \cup a_{2}$ are horizontal. Then $\alpha$ is said to connect parallel arcs if the orientations of $a_{1}, a_{2}$ points to the same direction; otherwise $\alpha$ connects antiparallel arcs. For example, if $F$ is a minimal Seifert surface then it is always $\pi_{1}$-injective, and $\alpha$ always connect antiparallel arcs. Set 
up a coordinate on the boundary of $B=N(\alpha)$ so that $a_{1} \cup a_{2}$ is a 0-tangle and $F \cap \partial B$ is isotopic to a $\frac{1}{0}$-tangle. Denote by $L(F, \alpha, r)$ the knot or link obtained from $L$ by replacing $a_{1} \cup a_{2}$ with a rational tangle $T_{r}$.

Corollary 6.9. Suppose $L$ is a non-split oriented link, $F$ is a $\pi_{1}$-injective spanning surface of $L$, and $\alpha$ an arc on $F$. Let $K=L(F, \alpha, 1 / n)$, where $|n|>2$ is odd if $\alpha$ connects parallel arcs, and even otherwise. If $K$ is a knot then it has a persistently laminar branched surface.

Proof. Let $B=N(\alpha)$ and $T_{1 / n}=K \cap B$, as defined above. If $n$ is odd, let $\gamma$ be an allowable path starting with the Delman channel in figure 10(b) followed by the upper boundary path, and if $n$ is even let $\gamma$ be the path in figure 14(a), except that the labels on the top vertex is $0 / 1$ and the labels at the bottom are $1 / 0,1 / 1, \ldots, 1 / n$ (so the diagram is now $\mathcal{D}(1 / n)$ reflected along a horizontal line). In the first case let $\Sigma(\gamma)$ be the branched surface constructed in Theorem 5.3. In the second case let $\Sigma(\gamma)$ be the one constructed in the proof of Theorem 6.6 and, as in the proof of Theorem 6.7 , we may reverse the orientation of the two left arcs of the tangle so that the orientation looks like that of $T_{r_{1}}$ in figure 14(d). The assumption that $n$ is odd if and only if $\alpha$ connects parallel arcs implies that the tangle can be rotated if necessary so that the orientation of the arcs on the boundary of the tangle defined by the Delman channel or half channel surface match those of $L-N(\alpha)$, so we can extend $\Sigma(\gamma)$ to a branched surface $\Sigma$ in the complement of $K$ by adding the tubes $Q$ around $L-\operatorname{Int} B$ and the surface $F-\operatorname{Int} B$, which is attached to $Q$ using the orientation of $L$ as its sink marks. By Lemma $3.7 \Sigma$ is pre-laminar. As before, the components of $E(\Sigma)$ inside of the ball $B=N(\alpha)$ are essential cusped manifolds, and the assumption that $F$ is $\pi_{1}$-injective implies that the outside component $E(\Sigma)$, which is the same as $E(L)$ cut along $F$, is also an essential cusped manifold. Therefore by Lemma $2.2 \Sigma$ is laminar. By construction $\Sigma$ has two meridional cusps, hence it remains laminar after all nontrivial Dehn surgery on $K$.

\section{Seifert fibered surgery on Montesinos knots}

Exceptional Dehn surgeries on arborescent knots have been determined except for atoroidal Seifert fibered surgeries on Montesinos knots of length 3 . The following is a result in this direction. 
Theorem 7.1. [12, Theorem 1.1.] Suppose $K=K\left(\frac{p_{1}}{q_{1}}, \frac{p_{2}}{q_{2}}, \frac{p_{3}}{q_{3}}\right)$ is a Montesinos knot of length 3 and $q_{i} \geq 2$. If $\frac{1}{q_{1}-1}+\frac{1}{q_{2}-1}+\frac{1}{q_{3}-1} \leq 1$ then $K$ admits no atoroidal Seifert fibered surgery.

Theorem 6.7 can be used to strengthen this result by adding restrictions to $p_{i}$. We separate two cases. Recall that a Montesinos knot $K$ of length 3 is a pretzel knot of length 3 if it can be written as $K\left(1 / q_{1}, 1 / q_{2}, 1 / q_{3}, n\right)$ for some integers $n$ and $\left|q_{i}\right| \geq 2$, and it is a genuine pretzel knot if in addition $n=0$.

Theorem 7.2. Let $K$ be a pretzel knot of length 3. If $K$ admits an atoroidal Seifert fibered surgery, then $K$ is equivalent to $K\left(\frac{1}{q_{1}}, \frac{1}{q_{2}}, \frac{1}{q_{3}}, n\right)$ such that either $n=0$ and hence $K$ is a genuine pretzel knot, or $n=-1$ and $q_{i} \geq 3$. In either case $q_{i}$ satisfy $\frac{1}{\left|q_{1}\right|-1}+\frac{1}{\left|q_{2}\right|-1}+\frac{1}{\left|q_{3}\right|-1}>1$.

Proof. The second part follows from Theorem 7.1, so we only need to prove the first part. By [1] $K(r)$ is not an atoroidal Seifert fibered manifold if it contains a genuine laminar branched surface, hence by Theorem 6.7 the result holds except that we may have $K=K\left(1 / q_{1}, 1 / q_{2}, p_{3} / q_{3},-1\right)$, where $q_{i} \geq 2$ and $p_{3}=1,2$ or $q_{3}-1$. We are done when $p_{3}=1$. Since $K$ is assumed to be a pretzel knot, the case $p_{3}=2$ does not occur unless $q_{3}=3$, in which case we also have $p_{3}=q_{3}-1$. If $p_{3}=q_{3}-1$ then $K=$ $K\left(1 / q_{1}, 1 / q_{2},\left(q_{3}-1\right) / q_{3},-1\right)=K\left(1 / q_{1}, 1 / q_{2}, 1 /\left(-q_{3}\right)\right)$, which is a genuine pretzel knot. If some $q_{i}=2$, say $i=1$, then $K\left(\frac{1}{2}, \frac{1}{q_{2}}, \frac{1}{q_{3}},-1\right)=K\left(-\frac{1}{2}, \frac{1}{q_{2}}, \frac{1}{q_{3}}\right)$ is a genuine pretzel knot; hence we may assume $q_{i} \geq 3$ when $n=-1$.

We now consider the case that $K=K\left(r_{1}, r_{2}, r_{3}\right)$ is not a pretzel knot. As in [12], we use $\bar{p}=\bar{p}(p, q)$ to denote the $\bmod q$ inverse of $-p$ with minimal absolute value, i.e., $\bar{p}$ satisfies $p \bar{p} \equiv-1 \bmod q$, and $2|\bar{p}| \leq q$. We can combine [12, Theorem 8.2] with Theorem 6.7 to obtain the following result for atoroidal Seifert fibered surgery on non-pretzel Montesinos knot.

Theorem 7.3. Let $K$ be a Montesinos knot of length 3. If $K$ is not a pretzel knot and $K$ admits an atoroidal Seifert fibered surgery $K(r)$, then $K$ is equivalent to one of the following:

(a) $K(-2 / 3,1 / 3,2 / 5)$;

(b) $K(-1 / 2,1 / 3,2 /(2 a+1))$ and $a \in\{3,4,5,6\}$;

(c) $K(-1 / 2,1 / q, 2 / 5)$ for some $q \geq 3$ odd.

Proof. By [12, Theorem 8.2], $K$ is equivalent to one of the following.

(1) $K\left(1 / 3, \pm 1 / 4, p_{3} / 5\right), p_{3} \equiv \pm 1 \bmod 5$; 
(2) $K\left(1 / 3, \pm 1 / 3, p_{3} / q_{3}\right),\left|\bar{p}_{3}\right| \leq 2$;

(3) $K\left(1 / 2,2 / 5, p_{3} / q_{3}\right), q_{3}=5$ or $7,\left|p_{3}\right|>1$;

(4) $K\left(1 / 2,1 / q_{2}, p_{3} / q_{3}\right), q_{2} \geq 5$ and $\left|\bar{p}_{3}\right| \leq 2$;

(5) $K\left(1 / 2,1 / 3, p_{3} / q_{3}\right),\left|\bar{p}_{3}\right| \leq 6$.

Since $K$ is not a pretzel knot, by Theorem 6.7 and [1] we have $K=K\left(1 / q_{1}\right.$, $\left.1 / q_{2}, 2 / q_{3},-1\right)$ with $q_{i}$ positive and $q_{3} \geq 5$, so it cannot be of type (1) or (3) above. We may write $q_{3}=2 a+1$, so $K=K\left(1 / q_{1}, 1 / q_{2}, 2 /(2 a+1),-1\right)$. Note that $a>1$ as otherwise $K$ would be a pretzel knot. Since $2 a \equiv-1 \bmod$ $q_{3}$, we have $\bar{p}_{3}=a$. Therefore if $K$ is in (2) or (4) then we have $\left|\bar{p}_{3}\right|=a=2$, so $q_{3}=5$ and $K$ is in conclusion (a) or (c). Finally if $K$ is of type (5) then $2 \leq a \leq 6$. When $a=2 K$ is in conclusion (c), and when $a=3,4,5,6$ it is in conclusion (b).

\section{Persistently laminar tangles}

Given a 2 -string tangle $(B, T)$, we can add another 2 -string tangle $\left(B_{1}, T_{1}\right)$ to it to make it a knot or link $K=T \cup T_{1}$ in $S^{3}=B \cup B_{1}$, called an extension of $T$. The gluing map $\varphi: \partial B \rightarrow \partial B_{1}$ is an orientation reversing map, sending a curve of slope $r$ on $\partial B$ to a curve of slope $-r$ on $\partial B_{1}$. The extension is $s$-non-trivial, or simply non-trivial when $s=0$, if there is no disk $D$ in $B_{1}$ separating the two strings of $T_{1}$, with $\partial D$ a slope $s$ curve on $\partial B$. It is a pretzel extension if $\left(B_{1}, T_{1}, s\right)$ can be isotoped so that $s$ is a horizontal loop on $\partial B_{1}$ and $T_{1}$ is a pair of vertical arcs.

Definition 8.1. A closed branched surface $\Sigma$ in $E(T)=B-\operatorname{Int} N(T)$ is persistently laminar with degeneracy slope $s$, if it is laminar in $K(r)$ for all $s$-non-trivial extensions $K$ of $T$ and all nontrivial slopes $r$ of $K$. In this case $(B, T)$ is called a persistently laminar tangle.

Brittenham [2] showed that the tangle $T(1 / 3,-1 / 3)$ is persistently laminar. It was proved by Youn [13] that the tangle $T(1 / 3,-1 / 5)$ is also persistently laminar. Using the techniques developed above, we can now construct many more persistently laminar branched surfaces. See Theorem 8.5 below.

Suppose $\Sigma$ is a branched surface in $E(T)$ for some tangle $(B, T)$. Denote by $E_{T}(\Sigma)=E(T)$ - Int $N(\Sigma)$ and call it the exterior of $\Sigma$ in the tangle space. A component of $E_{T}(\Sigma)$ is an outside component if it intersects $\partial E(T)$, otherwise it is an inside component. If the outside component $Y$ is a collar 
$\partial E(T) \times I$ of $\partial E(T)$ then we say a curve on $\partial Y-\partial E(T)$ has slope $r$ if it is isotopic in $\partial E(T) \times I$ to a curve of slope $r$ on $\partial B$.

Lemma 8.2. Let $(B, T)$ be a tangle, and let $\Sigma$ be a closed pre-laminar branched surface in $\operatorname{Int} E(T)$. If the inside components of $E_{T}(\Sigma)$ are essential cusped manifolds, and the outside component $Y$ of $E_{T}(\Sigma)$ is a collar of $\partial E(T)$ with a single cusp of slope $s$, then $\Sigma i$ s a persistently laminar branched surface with degeneracy slope $s$.

Proof. Let $\left(S^{3}, K\right)=(B, T) \cup\left(B_{1}, T_{1}\right)$ be an $s$-non-trivial extension of $(B, T)$ and consider $\Sigma$ as a branched surface in $S^{3}$. Then the exterior of $\Sigma$ is a union of a component $X$ which contains the knot $K$, and the inside components of $\Sigma$ in $B$, which by assumption are essential cusped manifolds. Since the outside component $Y$ of $E_{T}(\Sigma)$ is a collar of $\partial E(T)$ with a single cusp of slope $s$, we see that $X$ is the union of $B_{1}$ with two 1-handles $V_{1}, V_{2}$ (i.e., a regular neighborhood of $T$ in $B$ ) attached, and $K$ is the union of $T_{1}$ with the cores of $V_{i}$. The cusp on $\partial Y$ becomes a cusp $\gamma$ on $\partial X$ which is of slope $-s$ on $\partial B_{1}$. By assumption $\gamma$ does not bound a disk in $B_{1}-T_{1}$, which implies that it does not bound a disk in $X-K$.

By assumption $\Sigma$ is pre-laminar and the inside components of $\Sigma$ in $B$ are essential cusped manifold, therefore to prove that $\Sigma$ is a laminar branched surface in $K(r)$, by Lemma 2.2 it suffices to show that $X(r)$, the manifold obtained from $X$ by $r$ surgery on $K \subset X$, is an essential cusped manifold.

The frontier of $N\left(T_{1}\right)$ is a pair of annuli $A=A_{1} \cup A_{2}$ which cut $X$ into $E\left(T_{1}\right)$ and a solid torus $V$ with $K$ as its core. Since $K$ is a nontrivial extension, the curve $\gamma$ does not bound a disk in $V-K$, hence the surface $\partial E\left(T_{1}\right)-\gamma \cup A$, which is a union of two pairs of pants, is incompressible in $E\left(T_{1}\right)$, so $E\left(T_{1}\right)$ is an essential cusped manifold when considering $N(\gamma) \cup A$ as a vertical surface. After performing Dehn surgery on $K$ the solid torus $V$ becomes another solid torus $V(r)$ with $A$ as a pair of non-meridional annuli, hence it is also an essential cusped manifold when considering $A$ as vertical surface. It is easy to show that the union of essential cusped manifolds along vertical annuli is still an essential cusped manifold. Hence $X(r)=E\left(T_{1}\right) \cup V(r)$ is an essential cusped manifold.

Example 8.3. Consider the 2-complex $\Sigma=Q \cup P \cup D$ in figure 15, where $Q$ is a pair of tubes represented by the thick arcs, $P$ is a punctured sphere with two boundary components $a_{1}, a_{2}$ glued to two boundary components of $Q$ and the other two boundary components $b_{1}, b_{2}$ attached to the interior of $Q$, and $D$ is a union of two disks, one on each side of $P$. The sink marks are 


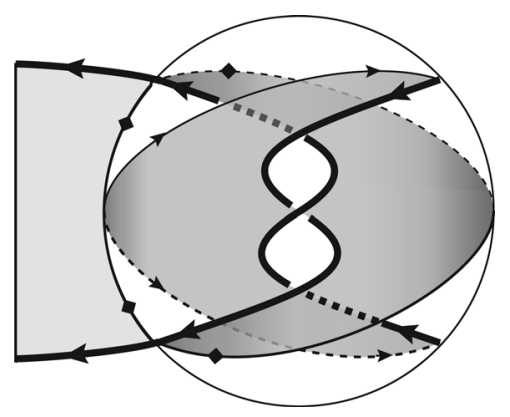

Figure 15

shown in the figure. Note that there is only one double points of $D$, which is of type IV, as shown in figure 5(a). The other singular points are on the two boundary components $b_{1}, b_{2}$ of $P$, which are of type (I) as in Definition 3.6. Therefore by Lemma 3.7 these sink marks can be extended to a branched surface structure for $\Sigma$.

The train track of $\partial \Sigma$ is positively oriented as shown in figure $7(\mathrm{a})$. One can check that the inside component of $E(\Sigma)$ is a solid torus with cusp winding number 2. The union of $\Sigma$ and its mirror image form a closed branched surface $\hat{\Sigma}$ in the exterior of $T(1 / 3,-1 / 3)$. By Lemma $3.7 \hat{\Sigma}$ is pre-laminar, and it is easy to see that it satisfies the conditions of Lemma 8.2. Therefore by that lemma $\hat{\Sigma}$ is a persistently laminar branched surface in $T(1 / 3,-1 / 3)$. It is slightly different from the one in [1].

It is important to note that the orientations of the sink marks make a big difference here. One can reverse the orientations of all oriented sink marks to obtain another branched surface with positively oriented boundary train track, but the inside component has cusp winding number 1 and hence is not an essential cusped manifold because it has a monogon, which is why $T(1 / 3,1 / 3)$ is not a persistently laminar tangle. Note that attaching a $T(-1 / 2)$ tangle to $T(1 / 3,1 / 3)$ makes a $(3,4)$ torus knot, which admits many lens space surgeries.

The above construction can be easily generalized. One can add more vertical crossings to obtain a branched surface $\Sigma(1 / q)$ for $T(1 / q)$ when $q>3$ is odd (but not if $q$ is even because the orientations will not match). One can show that the inside component of the exterior of $\Sigma(1 / q)$ is an essential cusped manifold, hence the union of $\Sigma\left(1 / q_{1}\right)$ and the mirror image of $\Sigma\left(1 / q_{2}\right)$ is a persistently laminar branched surface for $T\left(1 / q_{1},-1 / q_{2}\right)$ if $q_{1}, q_{2}$ are odd and $q_{i} \geq 3$. We can also replace the crossing in the middle by a horizontal 


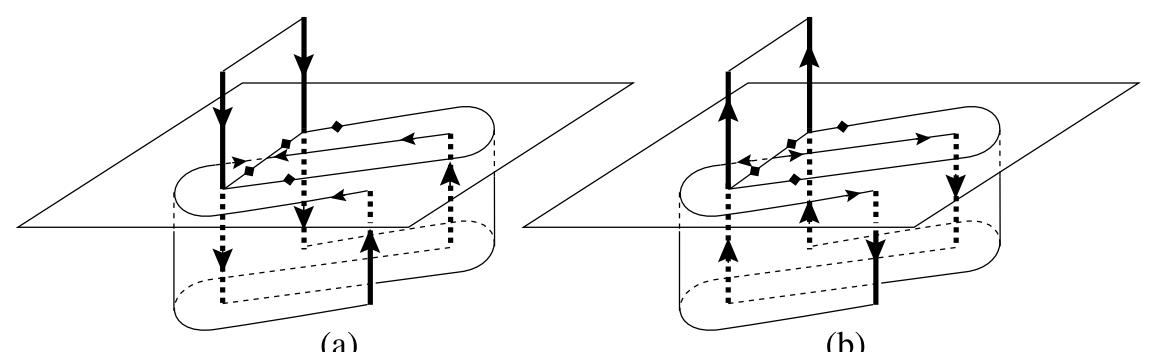

(a)

(b)

Figure 16

band with several crossings or other surfaces to create persistently laminar branched surfaces for more complicated tangles.

To get more general results, we need to modify Delman's channel surfaces. Let $e$ be the Delman channel of type A. Let $\Sigma^{\prime}(e)$ be the the branched surface obtained from the one in figure 9 (a) by deleting the upper half of the two tubes connected to the meridional cusps, as well as the disk attached to it. See figure 16(a). For the same reason as before, this is a pre-laminar branched surface, and the part below the horizontal sphere is the same as that of $\Sigma(e)$. Similarly, if $e$ is a Delman channel of type B then denote by $\Sigma^{\prime}(e)$ the branched surface shown in figure 16(b).

Given an allowable path $\gamma$ in $\mathcal{D}(p / q)$ starting with a Delman channel $e$, the branched surface $\Sigma(\gamma)$ starts with $\Sigma(e)$, which can be replaced by $\Sigma^{\prime}(e)$ above to obtain a new branched surface $\Sigma^{\prime}(\gamma)$. All the inside components of $E\left(\Sigma^{\prime}(\gamma)\right)$ are the same as those of $E(\Sigma(\gamma))$. The boundary train track of $\Sigma^{\prime}(\gamma)$ has only one component, which is negatively oriented if $e$ is of type A, and positively oriented if $e$ is of type B. The cusp on the outside of the top horizontal surface has slope $1 / 2$ in both cases.

Lemma 8.4. Suppose $r=p / q, q \geq 3$ is odd and $0<r<1$.

(1) If $\frac{1}{3}<r<1$ then $\mathcal{D}(r)$ has an allowable path $\gamma$ starting with a type $A$ Delman channel.

(2) If $0<r<\frac{2}{3}$ then $\mathcal{D}(r)$ has an allowable path $\gamma$ starting with a type $B$ Delman channel.

Proof. (1) If $\frac{1}{2}<r<1$ then $\mathcal{D}(r)$ is as shown in figure $17(\mathrm{a})$, in which case we can choose $\gamma$ to start with the type A channel followed by the lower 


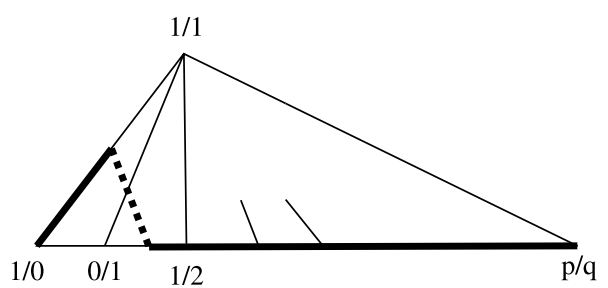

(a)

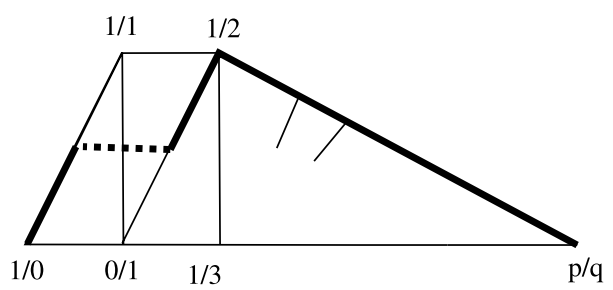

(b)

Figure 17

boundary path from $\frac{1}{2}$ to $\frac{p}{q}$, as shown in the figure. Similarly if $\frac{1}{3}<r<\frac{1}{2}$ then $\mathcal{D}(r)$ and $\gamma$ are as shown in figure 8.3(b).

(2) This is similar. In this case the diagram $\mathcal{D}(r)$ is obtained from that in figure 8.3 by reflecting along a horizontal line, then changing the label $p_{i} / q_{i}$ to $\left(q_{i}-p_{i}\right) / q_{i}$. The image of the path in the corresponding figure gives the $\gamma$ required.

Any Montesinos tangle of length 2 can be written as $T\left(r_{1},-r_{2}\right)$, where $r_{i}=p_{i} / q_{i}$. We can isotope it to one with $1 \leq p_{i}<q_{i}$. (This may change the degeneracy slope $s$.) Since $T\left(r_{1},-r_{2}\right)$ is homeomorphic to $T\left(r_{2},-\left(1-r_{1}\right)\right)$, we may also assume that $r_{1}+r_{2} \leq 1$. The following shows that many of these are persistent tangles if both $q_{i}$ are odd.

Theorem 8.5. If $0<r_{i}=p_{i} / q_{i}<\frac{2}{3}$ and $q_{i}$ are odd then $T=T\left(r_{1},-r_{2}\right)$ is persistently laminar.

Proof. Choose $\gamma_{i}$ to start with a type B Delman channel. Then $\Sigma_{i}=\Sigma^{\prime}\left(\sigma_{i}\right)$ is a branched surface with positively oriented boundary train track. The mirror image $-\Sigma_{2}$ of $\Sigma_{2}$ is a branched surface for $T\left(-r_{2}\right)$ with negatively oriented boundary train track. Let $\Sigma$ be obtained by gluing $\Sigma_{1}$ to $-\Sigma_{2}$ along their boundary. The cusps on $\Sigma_{i}$ are of slope $1 / 2$, so $\Sigma$ has a cusp of slope $1 / 2-1 / 2=0$ on the outside. By Lemma $8.2 \Sigma$ is a persistent laminar branched surface with degeneracy slope 0 .

Example 8.6. Suppose $r_{i}=p_{i} / q_{i}, 1 \leq p_{i} \leq q_{i}$ and $q_{i}$ are odd. Then the following tangles are all persistently laminar. (i) $T\left(1 / q_{1},-1 / q_{2}\right)$; (ii) $T\left(r_{1},-r_{1}\right)$; and (iii) $T\left(r_{1},-r_{2}\right)$ ) with $r_{2} \in\left(\frac{1}{3}, \frac{2}{3}\right)$. This follows from Theorem 8.5 for those in (i), as well as those in (ii) and (iii) when $r_{1}<\frac{2}{3}$. If $r_{1} \geq \frac{2}{3}$, we have $T\left(r_{1},-r_{1}\right)=T\left(r_{1}-1,1-r_{1}\right)=T\left(1-r_{1},-\left(1-r_{1}\right)\right)$, and $0<1-r_{1}<\frac{2}{3}$, 
so (ii) also follows from Theorem 8.5. Similarly if $r_{1} \geq \frac{2}{3}$ in case (iii), we have $T\left(r_{1},-r_{2}\right)=T\left(r_{1}-1,1-r_{2}\right)=T\left(1-r_{2},-\left(1-r_{1}\right)\right)$ and $1-r_{i} \leq \frac{2}{3}$, hence the result follows.

\section{Acknowledgment}

I would like to thank the referee for a careful reading and many helpful comments.

\section{References}

[1] M. Brittenham, Essential laminations in Seifert-fibered spaces, Topology 32 (1993), 61-85.

[2] M. Brittenham, Persistently laminar tangles, J. Knot Theory Ram. 8 (1999), 415-428.

[3] M. Brittenham and Y.-Q. Wu, The classification of exceptional Dehn surgeries on 2-bridge knots, Comm. Anal. Geom. 9 (2001), 97-113.

[4] C. Delman, Constructing essential laminations which survive all Dehn surgeries, preprint.

[5] D. Gabai, Problems in foliations and laminations, Geometric topology (Athens, GA, 1993), AMS/IP Studies Advance Mathematics 2.2 (1997), 1-33.

[6] D. Gabai and U. Oertel, Essential laminations in 3-manifolds, Ann. Math. 130 (1989), 41-73.

[7] A. Hatcher and W. Thurston, Incompressible surfaces in 2-bridge knot complements, Invent. Math. 79 (1985), 225-246.

[8] T. Li, Laminar Branched Surfaces in 3-manifolds, Geom. Topol. 6 (2002), 153-194.

[9] D. Rolfsen, Knots and links, Publish or Perish, 1990.

[10] Y.-Q. Wu, Dehn surgery on arborescent knots, J. Diff. Geom. 42 (1996), 171-197.

[11] Y.-Q. Wu, Sutured manifold hierarchies, essential laminations, and Dehn surgery, J. Diff. Geom. 48 (1998), 407-437. 
[12] Y.-Q. Wu, Immersed surfaces and Seifert fibered surgery on Montesinos knots, Trans. Amer. Math. Soc. (to appear).

[13] E.-J. Youn, Word hyperbolic Dehn surgery on Montesinos knots, University of Iowa Thesis, 2003.

Department of Mathematics

UNIVERSITY OF IOWA

IOWA CiTy

IA 52242, USA

E-mail address: wu@math.uiowa.edu

RECEIVED April 5, 2012 